\title{
General Universal Relativity (GURL): No Big-Bang
}

\author{
Bandula Dahanayake \\ Farmfield Crescent, Kanata, Canada \\ Email address: \\ Bandula_Dahanayake@yahoo.com
}

\section{To cite this article:}

Bandula Dahanayake. General Universal Relativity (GURL): No Big-Bang. International Journal of Astrophysics and Space Science. Vol. 4, No. 4, 2016, pp. 32-58. doi: 10.11648/j.ijass.20160404.11

Received: November 9, 2016; Accepted: December 6, 2016; Published: January 29, 2017

\begin{abstract}
General Universal Relativity (GURL) extends Universal Relativity (URL) on to accelerating frames. As with inertial frames, an accelerating body contracts in all directions resulting in mass density dilation, and hence the dilation of the surface gravity of the accelerating objects. Time and the mass of an accelerating object remain absolute. When acceleration is zero, GURL becomes URL. Light cannot travel forever in the visible frequency region without being succumb to a frequency shift due to the energy loss along the path. Visible universe is the maximum distance light can travel without being subjected to a frequency shift out of the visible region of the spectrum; it is not an indication that universe had a beginning. Visible universe is an observer dependent moving horizon; an indication that the universe is not finite. Cosmic microwave background is a result of distant light bursts that are frequency shifted out of the visible spectrum due to propagation loss. One person's cosmic microwave background is the visible universe for someone elsewhere and vice versa. Our cosmic microwave background carries the information about stars and galaxies beyond our visible region, but within someone else's visible region; this information does not represent the early universe. If we send a light burst, some of our out of the visible region neighbors will receive it in the microwave band. What we see in the distance is not our own past, but our distant neighbor's. No theoretical or observational basis to suggest universe is expanding; big-bang is not plausible. Galactic red shift is due to propagation loss; the further the galaxy away the higher the propagation loss and hence higher the red shift. Increasing galactic red shift indicates the radial movement of galaxies; it is not an indication of space expansion. Rates of change of masses of galaxies create orbits dilation or contraction giving the galaxies radial velocities proportional to the orbital distances. Any galaxy that is moving toward us, such as Andromeda, has a decreasing mass while the galaxies with increasing mass move away from us. A collision of earth with an asteroid would dilate the orbit of the earth abruptly bringing back the ice-age, making the earth un-inhabitable. Total amount of matter and energy in the universe is not calculable; there is no dark matter or dark energy. Although we are not at the center of the universe, we are at the center of a Local Galactic Cluster.
\end{abstract}

Keywords: Relativity, Light, Time, Mass, Space, Universe, Big-Bang, Cosmic-Microwave

\section{Introduction}

With the introduction of Maxwell's equations for the propagation of electromagnetic waves, one of the fundamental properties of the nature was revealed; the speed of light depends only on the electrical properties, permittivity and permeability of the medium. The speed of light is independent of the observer's frame of reference whether it is an inertial frame or accelerating frame [2]. Later, in 1905, Einstein used the Lorentz transformation $[2,10]$ to show that the path of light is relative, which we now know that it is not true [4]. In other words, under the assumption that the light is relative, if a light pulse of energy $e$ is fired vertically up from the bottom of a horizontally moving cabin of constant speed, the light pulse is assumed to take a vertical path relative to an observer inside the cabin just like what a vertically thrown golf ball would in a horizontally moving cabin of constant speed. If a light pulse of energy $e$ traveling at speed $c$, which is a universal constant determined by the electrical properties of the medium alone, is assumed to take a vertical path relative to the moving cabin, then, the light pulse must have and inertia and an equivalent mass purely due to the assumption that the light is relative, not as a fact of nature. Since the pulse of energy $e$ is travelling at speed $c$, the equivalent mass $m_{e q}$ by the assumption that the 
light is relative would be $m_{e q}=e / c^{2}$, which gave rise to the famous relationship $e=m c^{2}[2,3]$. It is also noteworthy that the equivalent mass $m_{e q}$ for electromagnetic energy that creeps out when the light is assumed to be relative, and the real mass $m$ of an object are not the same even though they are portrait to be the same in the $e=m c^{2}$ relationship.

Now, since the light pulse has an equivalent mass by the assumption that the light is relative, the behavior of the light pulse relative to the cabin supposed to be exactly the same as the behavior of a golf ball. Now the light pulse has a momentum, not as a fact of nature, but by assumption that the light is relative. The widely known view that the energy has a mass was resulted from the erroneous assumption that the light is relative, and the equally erroneous presumption that the kinetic energy and electromagnetic energy are the same (they are not the same). The idea that the mass and energy are one and the same is a result of this erroneous assumption that the light is relative, and the erroneous assumption that the kinetic energy is the same as the electromagnetic energy. If the light is not relative, then, the light pulse has no momentum or equivalent mass; mass and energy are not one and the same and $e \neq m c^{2}$. And also, since the kinetic energy is not the same as the electromagnetic energy, $e \neq m c^{2}$. There are more ways than one to show that the mass and energy are not one and the same or $e \neq m c^{2}$.

Since the light pulse has a momentum by the assumption that the light is relative, a stationary observer outside the cabin supposed to see that the light pulse is taking an angular path. This is because the light pulse has an equivalent electromagnetic mass under the assumption that the light is relative; so, with respect to a stationary observer outside the cabin, the pulse has a horizontal speed $u$, the speed of the cabin, and a vertical speed component $c$, that results in a longer angular path. In both those paths, the speed of the pulse remains the same, $c$. With reference to the observer inside the cabin, by assumption that the light is relative, the light pulse travels at speed $c$ vertically straight up and hit the ceiling. However, under the same assumption that the light is relative [1], with respect to the stationary observer outside, the light pulse travels a longer path at the same speed $c$ and hit the ceiling at an angle. How could this be? So, in order to explain this conundrum, an erroneous conclusion was drawn that the time must be relative and time must depend on the observer's frame of reference $[1,2]$. It is this misconception that led the way to the special relativity [2].

As it turns out, there are several problems with the Special Relativity $[4,5]$ :

i) The Lorentz-Einstein transformation $[1,2]$ the Special Relativity relies upon is not unique. There are infinitely many transforms that can transform the Maxwell's Equations on to an inertial frame [4]. This will result in an infinitely many relative times and infinitely many space-time functions for a given inertial frame.

ii) The relative time is directional [4]. The relative time along the direction of motion is different from the relative time on a lateral cross section perpendicular to the direction of motion.
Theorem: Universal Time and Mass

Time and mass are universal. Time and Mass are absolute. Time and Mass are Not Relative. Time and Mass do not depend on the observer's frame of reference

Proof: A Comprehensive proof can be found in [4]. Here we use a simple approach based on an argument. It is obvious that if the time and mass are relative, and depend on the observer's frame of reference, then, the relative time and relative mass have to be directional since the motion of any object is directional. Directional motions should always breed directional relative times and directional masses. However, the time and mass can't be directional. In order for relative time and relative mass to be non-directional, the speed of the object must be Omni-directional. On the other hand, an object with Omni-directional speed is an object at stand still. That means there is no such thing called relative time and relative mass. We only have time and mass that are universal; time and mass are absolute. A second is a second whether it is on earth or mars, or any other galaxy in the universe; it is the same with mass [4, 5].

Corollary: Time is not a fourth dimension one could travel on; you can travel neither forward nor backward in time. It is always the time that travels, not you. Time is a definition.

You just enjoy, suffer, complain or bear the ride. You have no choice. You can't turn time into a space dimension simply multiplying time, $t$ by the speed of light, $c$ and calling it a fourth dimension; the ct-axis only exists in your note book, not in the nature. Time is a definition, not a dimension.

iii). In the relationship $e=m c^{2}[1,3], e$ is electromagnetic energy, and $m c^{2}$ is kinetic energy. The electromagnetic energy and kinetic energy are two different forms of energy. They are not equal. They can be converted to each other by some means. If they had been equal we wouldn't have had any shortage of electric power. Even if the electromagnetic energy and the kinetic energy are the same, still the relationship $e=m c^{2}$ does not hold because LIGHT IS NOT RELATIVE [4, 5]. A vertical light pulse from the bottom of a horizontally moving cabin of constant speed does not take a straight vertical path relative to an observer inside the cabin. A light pulse has NO momentum, and hence, the light pulse has no equivalent mass. Quite simply, $e \neq m c^{2}$. A pulse of light does not behave like a golf ball. Mass and electromagnetic energy are not one and the same [4, 5].

iv). If the light is relative, it results in Shear Electromagnetic (SEM) waves that travel at a speed that depends on the speed of the frame of reference [4]. Only when the speed of the object reaches the speed of the Transverse Electromagnetic (TEM) waves, $c$, the speed of the Shear Electromagnetic (SEM) waves reaches speed $c$. So, if the light is relative, the speed of light can no longer be considered a constant that depend only on the electrical properties of the medium. The speed of the SEM waves, $c_{s}$ is given by, $c_{s}=(1-\eta) c^{2} / u$ where, $\eta=\left[1-u^{2} / c^{2}\right]^{1 / 2}, u$ is the speed of the frame of reference, $c$ is the speed of TEM waves.

And the fact of the matter is that there is no such thing 
called SEM waves in the nature. The SEM waves only come into a theoretical existence if the light is relative [4]. The absence of Shear Electromagnetic (SEM) waves in nature is a good testimony to the fact that the light is not relative. If the light is relative, there must exist SEM waves in the nature, and the speed of light is observer dependent.

Since there is no space-time [4], the General Relativity does not hold true. In the absence of General relativity, there are no worm holes or pot holes in the space. Further, in the absence of General Relativity, there is/was no big-bang either. The universe has no beginning; without a beginning, an end cannot exist either. So, there is no end to the universe; for an end to exist there must be a beginning. Whatever that doesn't have a beginning never ends. The idea that the universe is 13.5 billion years old came out of the Hubble relationship and the assumption that the universe is expanding; this idea has no merit because there is no expansion of the universe [7]. If you use the inverse of the Hubble constant, as it is done $[9,10]$, to obtain the age of the universe, the age of the universe will be a constant; forever young. Age can't be a constant. The derivation of the age of the universe is simply incorrect and the big bang is not plausible.

Light is Not Relative

The light does not travel relative to a source $[4,5,6]$. A source of light releases light as electromagnetic wave bursts of constant duration; the duration of a burst of light $F$ is a universal constant, where $\mathrm{F}=h / e, h$ is the Plank constant and $e$ is the quantum energy, the smallest energy level that exist in the nature. A light burst of frequency ne/h has energy ne, where $n=1,2, \ldots$. Once a wave burst is out of a source, the source has nothing to do with the wave burst any longer; the path of light is completely determined by the density gradient of the medium. The light follows the density gradient of the medium; in other words, light follows the curvature of the medium. How far a burst of light can travel is determined by the path energy loss; a burst of light cannot travel forever. As a burst of light travels further and further, it loses its energy due to path energy loss resulting in a frequency drift that will slowly moves the burst away from the visible region of the spectrum, and finally to the total fading away. The special relativity does not hold true in the nature since the light is not relative.

As it was shown in the Universal Relativity $[4,5]$, the time is absolute. The time does not depend on the frame of reference. The mass of an object does not depend on the frame of reference. The electromagnetic energy does not have momentum, inertia. When an object is in motion, the time and mass remain absolute. However, the geometry of a moving object shrinks in all directions, which results in a contraction of the volume of a moving object. The shrinkage of the volume is a function of the speed of the object. This volume contraction of a moving object gives rise to the dilation of the surface gravity of a moving object. When the speed of the moving object reaches the speed of the light $c$, the volume of the object approaches zero and the surface gravity of the object reaches infinity [5]; this sets the speed limit of any object in universe to the speed of light $c[5,2]$.
The Real Moving Cabin Scenario:

What is the scenario of moving cabin when the light is not relative? The cabin is moving horizontally at a uniform speed. When a light pulse is fired inside the cabin vertically, relative to the observer in the cabin, the light pulse is moving away from the observer at the speed of the cabin. At the same time, relative to the observer inside the cabin, the light pulse is also moving vertically at the speed $c$. Therefore, with relative to the observer inside the cabin, the light pulse takes a longer angular path. When the light is not relative, as it is an actual fact in the nature, the path of light relative to an observer inside the cabin is angular. A stationary observer outside the cabin sees that the light pulse is taking a straight vertical path. The path light takes has nothing to do with the speed of the source; once the light is out of the source, the path light takes is determined by the medium alone, nothing else. This is complete opposite of what happens when a golf ball is used in place of the light pulse. A golf ball has a momentum; the light pulse does not [5].

Now, we know what happens when an object is moving at a constant speed. We want to know what happens when an object is accelerating. How does the constancy of the speed of light play out in the case of accelerating frames? How can we extend the Universal Relativity (URL) $[5,4]$ from an inertial frame to an accelerating frame? In other words, how can we generalize the Universal Relativity (URL) to incorporate the accelerating frames? If we do that, we can obtain the Universal Relativity for inertial frames as a special case of the General Universal Relativity (GURL) for accelerating frames when acceleration $\alpha=0$.

Non-Expanding Universe:

Let us assume that a cave-man that had been living his whole life inside a cave wondered out of his burrow for the first time and sees an object in the sky at a distance moving towards him. His first reaction is quite clear. The sky is falling! If the object is moving away from him, he would have said "the sky is expanding". Could this scenario be any different from our saying today that "the universe is expanding?" We peek into distance galaxies and see them moving away from us and declare that "the space is expanding". Just because we see the nearby galaxies are moving away $[8,10]$, to say that the universe is expanding is simply unrealistic.

In order to find a theoretical basis for our observation that some galaxies are moving away from us while some other galaxies are moving toward us, we use the Local Galactic Cluster Model (LGCM) where nearby galaxies are orbiting around one central galaxy in the cluster to form a Local Galactic Cluster (LGC). In our Local Galactic Cluster, the nearby galaxies are orbiting around the Milky Way galaxy. Several nearby Local Galactic Clusters orbit around one central Local Galactic Cluster to form a Local Super Galactic Cluster (LSGC), and so on the process continues onward to form bigger and bigger clusters in the universe. The Local Galactic Cluster dynamics, which explain why our nearby galaxies are moving away from us at an accelerated rate, are derived.

In the Local Galactic Clusters (LGC) presented here, 
orbiting center of each Galactic Cluster is also a galaxy at the center of the cluster; the rest of the galaxies in the Local Galactic Cluster are orbiting around the galaxy at the orbiting center. In our Local Galactic Cluster the orbiting center is our Milky Way Galaxy.

\section{The Model}

Consider a cylindrical cabin moving along a horizontal axis at acceleration $\alpha$. Then, the speed of the cabin at any time $t$ is given by,

$$
\mathrm{v}(\mathrm{t})=\mathrm{u}+\alpha \mathrm{t}
$$

where $u$ is the inertial speed or the constant steady state speed.

When the acceleration $\alpha=0$, we have an inertial frame moving at a constant speed $u$.

At time $t$, with respect to an observer inside the cabin, the length of the cabin along the direction of motion is $\ell(t)$ and the diameter of the cabin $h(t)$. When the cabin is at stand still, that is when $v(t)=0$, the length of the cabin is $L$ and the diameter of the cabin is $H$.
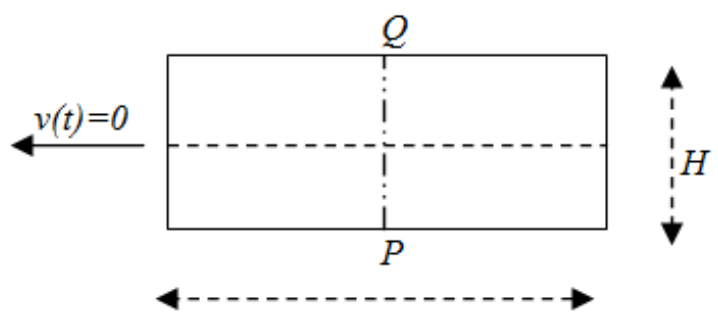

$\mathrm{L}$

Figure 1. Stand Still Cabin (Not Moving).

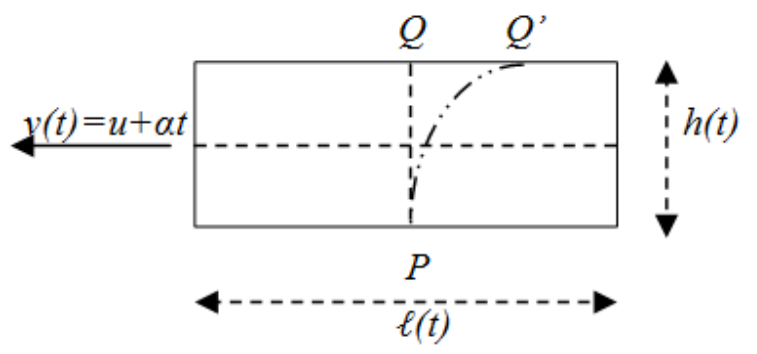

Figure 2. Accelerating Cabin (Moving).

There are observers both inside the cabin and the outside the cabin. We want to see what happen to an accelerating object as a result of its acceleration.

\section{The General Universal Relativity (GURL)}

From the Universal Relativity (URL), we already know what happens to a moving body when the body moves at constant speed; the moving body contracts in all directions while time and the mass remain absolute. Our aim is to find out the effect of acceleration on a moving body. We want to find out what happen to the longitudinal dimension along the direction of motion, and also what happen to the lateral dimensions on the plane perpendicular to the direction of motion. We already know that the time and mass are absolute; the time and mass remain the same whether an object is at standstill or moving. When we say time remains the same, we mean that a second is a second everywhere in the universe irrespective of the frame of reference.

Theorem-3.1: General Lateral Contraction

When an object is in an accelerated motion, its lateral dimensions, perpendicular to the direction of motion, contract. The lateral dimension $h(t)$ at time $t$ is given by, $\mathrm{h}(\mathrm{t})=\mathrm{n}(\mathrm{t}) \mathrm{H}$,

where, $\eta(t)=\left[1-\beta(t)(u / c)^{2}\right]^{1 / 2}, u$ is the inertial speed, $\alpha$ is the acceleration,

$$
\beta(t)=1+(\alpha t / u)[2+H / c t]+(\alpha t / u)^{2}\left[1+H / c t+\frac{1}{3}(H / c t)^{2}\right],
$$

$H$ is the lateral dimension perpendicular to direction of motion of the object at stand still, $\alpha$ is the acceleration, $u$ is the inertial speed, $c$ is the speed of light, and $t$ is the time.

The proof is given in Appendix-A.

Corollary-3.1: Approximate General Lateral Contraction

When an object is at an accelerated motion, its lateral dimensions, perpendicular to the direction of motion, contract. The approximate lateral dimension $h(t)$ at time $t$ is given by, $h(t)=n(t) H$, where, $\mathrm{\eta}(\mathrm{t})=\left[1-(\mathrm{v} / \mathrm{c})^{2}\right]^{1 / 2}$,

$v=u+\alpha t, u$ is the inertial speed, $\alpha$ is the acceleration,

Proof: From General Lateral Contraction Theorem-3.1, we have, $\mathrm{h}(\mathrm{t})=\eta(\mathrm{t}) \mathrm{H}$, where, $\eta(\mathrm{t})=\left[1-\beta(\mathrm{t})(\mathrm{u} / \mathrm{c})^{2}\right]^{1 / 2}, u$ is the inertial speed, $\alpha$ is the acceleration,

$$
\beta(t)=1+(\alpha t / u)[2+H / c t]+(\alpha t / u)^{2}\left[1+H / c t+\frac{1}{3}(H / c t)^{2}\right],
$$

As time passes, $\mathrm{H}<<$ ct, $2+\mathrm{H} / \mathrm{ct} \cong 2$ and,

$$
\begin{gathered}
\left.1+\mathrm{H} / \mathrm{ct}+\frac{1}{3}(\mathrm{H} / \mathrm{ct})^{2}\right] \cong 1 \text {, hence, } \beta(\mathrm{t})=1+2 \alpha \mathrm{t} / \mathrm{u}+(\alpha \mathrm{t} / \mathrm{u})^{2}, \\
\beta(\mathrm{t})=[(\mathrm{u}+\alpha \mathrm{t}) / \mathrm{u}]^{2}, \beta(\mathrm{t})=(\mathrm{v} / \mathrm{u})^{2}
\end{gathered}
$$

Substituting for $\beta(t)$ in $\eta(t)$, we get, $\mathrm{n}(\mathrm{t})=\left[1-(\mathrm{v} / \mathrm{c})^{2}\right]^{1 / 2}$, where, $v=u+\alpha t$.

So, the Universal Relativity is not limited to inertial frames. It applies to accelerating frames. The lateral dimension contraction of an accelerating frame at time $t$ can be obtained by substituting instantaneous speed in the Universal Relativity formulation [5].

Lemma-3.1: Inertial Lateral Contraction

The lateral dimension contraction for an inertial frame of speed $u$ is given by, $\mathrm{h}=\left[1-(\mathrm{u} / \mathrm{c})^{2}\right]^{1 / 2} \mathrm{H}$

Proof: From the General Lateral Contraction Theorem-3.1, we have, $\mathrm{h}(\mathrm{t})=\mathrm{n}(\mathrm{t}) \mathrm{H}$, where, $\mathrm{n}(\mathrm{t})=\left[1-\beta(\mathrm{t})(\mathrm{u} / \mathrm{c})^{2}\right]^{1 / 2}, u$ is the inertial speed, $\alpha$ is the acceleration,

$$
\beta(t)=1+(\alpha t / u)[2+H / c t]+(\alpha t / u)^{2}\left[1+H / c t+\frac{1}{3}(H / c t)^{2}\right],
$$

Since, $\lim _{\alpha=0} \beta(t)=1$ at any time $t, \mathrm{n}(\mathrm{t})=\left[1-(\mathrm{u} / \mathrm{c})^{2}\right]^{1 / 2}$, and it is independent of $t$. So, we have, $\mathrm{h}=\mathrm{nH}, \mathrm{\eta}=\left[1-(\mathrm{u} / \mathrm{c})^{2}\right]^{1 / 2}$

We can obtain the same result by using the approximate lateral contraction in Corollary-1 when $\alpha=0$. It is also clear 
that we can use the General Lateral Contraction Theorem to obtain the Lateral Contraction Theorem for an inertial frame of speed $u$. This is exactly the same result obtained for inertial frames of speed $u$ in the Universal Relativity [5].

Theorem-3.2: General Lateral Cross Section Contraction

When an object is at an accelerated motion, its cross section contracts, and the cross section area $A(t)$ at time $\mathrm{t}$ is given by, $A(t)=\eta^{2}(t) A_{o}$, where $n(t)=\left[1-\beta(t)(u / c)^{2}\right]^{1 / 2}$

$$
\beta(\mathrm{t})=1+(\alpha \mathrm{t} / \mathrm{u})[2+\mathrm{H} / \mathrm{ct}]+(\alpha \mathrm{t} / \mathrm{u})^{2}\left[1+\mathrm{H} / \mathrm{ct}+\frac{1}{3}(\mathrm{H} / \mathrm{ct})^{2}\right]
$$

$\alpha$ is the acceleration, $A_{o}$ is the cross section area at stand still, $u$ is the inertial speed, $H$ is the diameter of the cylinder at stand still, $c$ is the speed of light, and $t$ is the time.

Proof: From the General Lateral Contraction Theorem-3.1, the diameter $h(t)$ of the cabin at time $t$ is given by,

$\mathrm{h}(\mathrm{t})=\mathrm{n}(\mathrm{t}) \mathrm{H}$. The cross section are $A(t)$ is given by,

$$
\mathrm{A}(\mathrm{t})=\pi\left(\frac{1}{2} \mathrm{~h}(\mathrm{t})\right)^{2} \text {, i.e., } \mathrm{A}(\mathrm{t})=\pi\left(\frac{1}{2} \mathrm{n}(\mathrm{t}) \mathrm{H}\right)^{2} \text {. Let } \mathrm{A}_{\mathrm{o}}=\pi\left(\frac{1}{2} \mathrm{H}\right)^{2},
$$

where $A_{o}$ is the cross section area at stand still. So, we have, $\mathrm{A}(\mathrm{t})=\mathrm{n}^{2}(\mathrm{t}) \mathrm{A}_{\mathrm{o}}$.

Further, $\lim _{a=0} \beta=1$, hence $\mathrm{n}(\mathrm{t})=\left[1-(\mathrm{u} / \mathrm{c})^{2}\right]^{1 / 2}$, which is independent of time $t$.

Corollary-3.2: Approximate General Lateral Cross Section Contraction

When an object is at an accelerated motion, its cross section contracts, and the cross section area $A(t)$ at time $\mathrm{t}$ is given by, $\mathrm{A}(\mathrm{t})=\left[1-(\mathrm{v} / \mathrm{c})^{2}\right] \mathrm{A}_{0}$, where $v=u+a t, \quad \alpha$ is the acceleration, $A_{o}$ is the cross section area at stand still, $u$ is the inertial speed, $c$ is the speed of light, and $t$ is the time.

Proof: From Approximate General Lateral Contraction, Corollary-3.1, we have the approximate $\eta(t)$,

$\eta(\mathrm{t})=\left[1-(\mathrm{v} / \mathrm{c})^{2}\right]^{1 / 2}$, where, $v=u+\alpha t, u$ is the inertial speed, $\alpha$ is the acceleration, From the General Lateral Cross Section Contraction Theorem-3.2, we have, $\mathrm{A}(\mathrm{t})=\mathrm{n}^{2}(\mathrm{t}) \mathrm{A}_{\mathrm{o}}$, So, we have, $\mathrm{A}(\mathrm{t})=\left[1-(\mathrm{v} / \mathrm{c})^{2}\right] \mathrm{A}_{\mathrm{o}}$

Lemma-3.2: Inertial Lateral Cross Section Contraction

When the acceleration is zero, the cross section contraction of an inertial frame of speed $u$ is independent of $t$ and it is given by, $\mathrm{A}(\mathrm{t})=\left[1-(\mathrm{u} / \mathrm{c})^{2}\right] \mathrm{A}_{\mathrm{o}}$.

Proof: The cross section area of an acceleration body is given by, $A(t)=\eta(t)^{2} A_{o}$, where $\eta(t)=\left[1-\beta(t)(u / c)^{2}\right]^{1 / 2}$.

We have, $\lim _{\alpha=0} \beta(t)=1$. Hence, $\lim _{\alpha=0} \eta(t)=\left[1-(\mathrm{u} / \mathrm{c})^{2}\right]^{1 / 2}$.

So, we have, $A(t)=\left[1-(\mathrm{u} / \mathrm{c})^{2}\right] \mathrm{A}_{\mathrm{o}}$.

We get the same result by substituting $\alpha=0$ in the Approximate General Cross Section Contraction given in Corollary-3.2. This is exactly the same result obtained for inertial frames of speed $u$ in the Universal Relativity [5].

Theorem-3.3: General Longitudinal Contraction

When an object is in an accelerated motion, its longitudinal dimension along the direction of motion, contracts. The longitudinal dimension $\ell(t)$ at time $t$ is given by, $\ell(\mathrm{t})=\left[1-(\mathrm{v} / \mathrm{c})^{2}\right] \gamma(\mathrm{t}) \mathrm{L}$, where

$$
\begin{gathered}
\gamma(\mathrm{t})=\varphi\left\{1-\frac{1}{2} \varphi\left[\alpha \mathrm{L}(\mathrm{c}+\mathrm{v}) /(\mathrm{c}-\mathrm{v}) \mathrm{c}^{2}\right]\right\} \\
\varphi=1+\alpha \mathrm{L} / \mathrm{c}(\mathrm{c}+\mathrm{v})
\end{gathered}
$$

$v=u+\alpha t, u$ is the inertial speed, $\alpha$ is the acceleration, and $t$ is the time.

The proof is given in Appendix-B.

Corollary-3.3: Approximate General Longitudinal Contraction

When an object is in an accelerated motion, its longitudinal dimension along the direction of motion, contracts. The longitudinal dimension $\ell(t)$ at time $t$ is given by, $\ell(\mathrm{t})=\left[1-(\mathrm{v} / \mathrm{c})^{2}\right] \mathrm{L}$, where $\mathrm{v}$ is the instantaneous speed at time $t$ given by, $v=u+\alpha t, u$ is the inertial speed, $\alpha$ is the acceleration.

Proof: From General Longitudinal Contraction Theorem-3.3, we have, $\ell(\mathrm{t})=\left[1-(\mathrm{v} / \mathrm{c})^{2}\right] \gamma(\mathrm{t}) \mathrm{L}$, where

$$
\begin{gathered}
\gamma(\mathrm{t})=\varphi\left\{1-\frac{1}{2} \varphi\left[\alpha \mathrm{L}(\mathrm{c}+\mathrm{v}) /(\mathrm{c}-\mathrm{v}) \mathrm{c}^{2}\right]\right\} \\
\varphi=1+\alpha \mathrm{L} / \mathrm{c}(\mathrm{c}+\mathrm{v})
\end{gathered}
$$

$v=u+\alpha t, u$ is the inertial speed, $\alpha$ is the acceleration, and $t$ is the time.

$$
\begin{aligned}
{\left[1-(\mathrm{v} / \mathrm{c})^{2}\right] \gamma(\mathrm{t})=\varphi } & \left\{\left[1-(\mathrm{v} / \mathrm{c})^{2}\right]-\frac{1}{2} \varphi\left[\alpha \mathrm{L}(\mathrm{c}+\mathrm{v}) /(\mathrm{c}-\mathrm{v}) \mathrm{c}^{2}\right]\left[(\mathrm{c}+\mathrm{v})(\mathrm{c}-\mathrm{v}) / \mathrm{c}^{2}\right]\right\} \\
& =\varphi\left\{\left[1-(\mathrm{v} / \mathrm{c})^{2}\right]-\frac{1}{2} \varphi\left[\alpha \mathrm{L}(\mathrm{c}+\mathrm{v})^{2} / \mathrm{c}^{4}\right]\right\}
\end{aligned}
$$

We know $\alpha L / c^{2}$ is a constant for a give object at given acceleration; it doesn't change with time. Further, $\alpha \mathrm{L} / \mathrm{c}^{2}<<1$, hence, $\varphi \cong 1$ and $\left[1-(\mathrm{v} / \mathrm{c})^{2}\right]-\frac{1}{2} \varphi\left[\alpha \mathrm{L}(\mathrm{c}+\mathrm{v})^{2} / \mathrm{c}^{4}\right] \cong 1-(\mathrm{v} / \mathrm{c})^{2}$.

So, $\left[1-(\mathrm{v} / \mathrm{c})^{2}\right] \gamma(\mathrm{t}) \cong\left[1-(\mathrm{v} / \mathrm{c})^{2}\right]$, and hence, $\ell(\mathrm{t})=\left[1-(\mathrm{v} / \mathrm{c})^{2}\right] \mathrm{L}$ where $v=u+\alpha t$.

The contraction of an accelerating frame at time $t$ can be obtained by substituting instantaneous speed in the Universal Relativity (URL) formulation [5].

So, we can use the Universal Relativity (URL) for an inertial frame to obtain General Universal Relativity (GURL) for an accelerating object. All we have to do is substitute the instantaneous speed of the object at time $t$ in place of the inertial constant speed in the Universal Relativity [5]; the outcome is GURL.

Lemma-3.3: Inertial Longitudinal Contraction

When the acceleration zero, the Longitudinal Contraction of an inertia frame of speed $u$ is given by,

$$
\ell=\left[1-(\mathrm{u} / \mathrm{c})^{2}\right] \mathrm{L}
$$

Proof: From the General Longitudinal Contraction Theorem-3.3, we have, $\ell(\mathrm{t})=\left[1-(\mathrm{v} / \mathrm{c})^{2}\right] \gamma(\mathrm{t}) \mathrm{L}$, where

$$
\begin{gathered}
\gamma(\mathrm{t})=\varphi\left\{1-\frac{1}{2} \varphi\left[\alpha \mathrm{L}(\mathrm{c}+\mathrm{v}) /(\mathrm{c}-\mathrm{v}) \mathrm{c}^{2}\right]\right\} \\
\varphi=1+\alpha \mathrm{L} / \mathrm{c}(\mathrm{c}+\mathrm{v}) \\
v=u+\alpha t .
\end{gathered}
$$

We know that, $\lim _{\alpha=0} \gamma(t)=1$, and $\lim _{\alpha=0} \mathrm{v}=u$.

Therefore, we have, $\ell=\left[1-(\mathrm{u} / \mathrm{c})^{2}\right] \mathrm{L}$.

We can obtain the same result by using the approximate longitudinal contraction in Corollary-3.3 when $\alpha=0$. This is also exactly the same result we had in the Universal Relativity. The inertial frame contraction is independent of time $t$. It is also clear that we can use the General 
Longitudinal Contraction Theorem to obtain the Longitudinal Contraction Theorem for an inertial frame of speed $u$ [5].

Theorem-3.4: General Volume Contraction

When an object is accelerating, its volume contracts, and the volume $V(t)$, at time $t$ is given by,

$$
\begin{gathered}
\mathrm{V}(\mathrm{t})=\left[1-\beta(\mathrm{t})(\mathrm{u} / \mathrm{c})^{2}\right]\left[1-(\mathrm{v} / \mathrm{c})^{2}\right] \gamma(\mathrm{t}) \mathrm{V}_{\mathrm{o}}, \text { where, } \mathrm{v}=\mathrm{u}+\alpha \mathrm{t} \\
\beta(\mathrm{t})=\left\{1+(\alpha \mathrm{t} / \mathrm{u})[2+\mathrm{H} / \mathrm{ct}]+(\alpha \mathrm{t} / \mathrm{u})^{2}\left[1+\mathrm{H} / \mathrm{ct}+\frac{1}{3}(\mathrm{H} / \mathrm{ct})^{2}\right]\right\} \\
\gamma(\mathrm{t})=\varphi\left\{1-\frac{1}{2} \varphi\left[\alpha \mathrm{L}(\mathrm{c}+\mathrm{v}) /(\mathrm{c}-\mathrm{v}) \mathrm{c}^{2}\right]\right\} \\
\varphi=1+\alpha \mathrm{L} / \mathrm{c}(\mathrm{c}+\mathrm{v})
\end{gathered}
$$

$\alpha$ is the acceleration, $H$ is the diameter of the body at stand still, $L$ is the length of the cylinder at stand still, $V_{o}$ is the volume stand still, $c$ is the speed of light, and $t$ is the time.

Proof: The cross section area at time $t$ is, $\mathrm{A}(\mathrm{t})=\eta^{2}(\mathrm{t}) \mathrm{A}_{\mathrm{o}}$ where $\eta(\mathrm{t})=\left[1-\beta(\mathrm{t})(\mathrm{u} / \mathrm{c})^{2}\right]^{1 / 2}$. The length at time $t$ is given by, $\ell(\mathrm{t})=\left[1-(\mathrm{v} / \mathrm{c})^{2}\right] \gamma(\mathrm{t}) \mathrm{L}$, where

$$
\begin{gathered}
\gamma(\mathrm{t})=\varphi\left\{1-\frac{1}{2} \varphi\left[\alpha \mathrm{L}(\mathrm{c}+\mathrm{v}) /(\mathrm{c}-\mathrm{v}) \mathrm{c}^{2}\right]\right\} \\
\varphi=1+\alpha \mathrm{L} / \mathrm{c}(\mathrm{c}+\mathrm{v}) \\
v=u+\alpha t
\end{gathered}
$$

So the volume at time $t$ can be obtained as,

$$
\mathrm{V}(\mathrm{t})=\mathrm{A}(\mathrm{t}) \ell(\mathrm{t})=\left[1-\beta(\mathrm{t})(\mathrm{u} / \mathrm{c})^{2}\right]\left[1-(\mathrm{v} / \mathrm{c})^{2}\right] \gamma(\mathrm{t}) \mathrm{A}_{\mathrm{o}} \mathrm{L}
$$

Since $V_{o}=A_{o} L$, the volume at stand still, we have

$$
\mathrm{V}(\mathrm{t})=\left[1-\beta(\mathrm{t})(\mathrm{u} / \mathrm{c})^{2}\right]\left[1-(\mathrm{v} / \mathrm{c})^{2}\right] \gamma(\mathrm{t}) \mathrm{V}_{\mathrm{o}}
$$

Corollary-3.4: Approximate General Volume Contraction

When an object is accelerating, its volume contracts, and the volume $V(t)$, at time $t$ is given by,

$\mathrm{V}(\mathrm{t})=\left[1-(\mathrm{v} / \mathrm{c})^{2}\right]^{2} \mathrm{~V}_{\mathrm{o}}$ where $v=u+\alpha t, V_{o}$ is the volume stand still.

Proof: From Corollaty-3.2, and Corollary-3.3, we have approximate cross section $A(t)$ and the approximate longitudinal dimension, $\ell(t)$,

$$
\begin{gathered}
A(t)=\left[1-(v / c)^{2}\right] A_{o}, \\
\ell(t)=\left[1-(v / c)^{2}\right] L .
\end{gathered}
$$

We also have, $\mathrm{V}_{\mathrm{o}}=\mathrm{A}_{\mathrm{o}} \mathrm{L}$.

So, the Approximate General Volume Contraction $V(t)$ is given by,

$$
\begin{gathered}
\mathrm{V}(\mathrm{t})=\mathrm{A}(\mathrm{t}) \ell(\mathrm{t}), \\
\mathrm{V}(\mathrm{t})=\left[1-(\mathrm{v} / \mathrm{c})^{2}\right]^{2} \mathrm{~V}_{\mathrm{o}},
\end{gathered}
$$

where $v=u+\alpha t$.

Lemma-3.4: Inertial Volume Contraction

When the acceleration is zero, the volume of a moving object at constant speed is given by,

$$
\mathrm{V}=\left[1-(\mathrm{u} / \mathrm{c})^{2}\right]^{2} \mathrm{~V}_{\mathrm{o}}
$$

where $u$ is the speed of the moving object and $V_{o}$ is the volume at stand still

Proof: From the volume the accelerating object, we have,

$$
\mathrm{V}(\mathrm{t})=\mathrm{V}_{\mathrm{o}}\left[1-\beta(\mathrm{t})(\mathrm{u} / \mathrm{c})^{2}\right]\left[1-(\mathrm{v} / \mathrm{c})^{2}\right] \gamma(\mathrm{t}) .
$$

We know that the $\lim _{\alpha=0} \beta(t)=1, \lim _{\alpha=0} \gamma(t)=1$, and $\lim _{\alpha=0} v=u$, So, we get,

$$
\mathrm{V}=\left[1-(\mathrm{u} / \mathrm{c})^{2}\right]^{2} \mathrm{~V}_{\mathrm{o}}
$$

We can obtain the same result by using the Approximate General Volume Contraction given in Corollary-3.4 when $\alpha=0$. This is exactly the same result we had in the Universal Relativity [5]. The inertial frame volume contraction is independent of time $t$.

\section{Transient Black Holes}

As the speed of an object increases, its volume decreases. When the volume reaches zero, its mass density becomes infinite while the mass remains unchanged, or absolute. In other words, at certain finite time, an accelerating object turns itself into a black hole [5].

Theorem-4.1: Accelerating Transient Black hole

Any accelerating body will turn itself into a black hole at time $t$, when, $\mathrm{t}=(\mathrm{c}-\mathrm{u}) / \alpha$, where, $u$ is the inertial speed, $\alpha$ is the acceleration, $\alpha \neq 0$, and $c$ is the speed of light.

Proof: Let the mass the accelerating body be $m$, then the mass density $\rho(t)$ at time $t$ is given by,

$$
\rho(t)=m / V(t) .
$$

The mass is absolute, and hence remains the same irrespective of the movement of the object. The mass of an object remains the same under acceleration. However, since the volume of an accelerating body contracts with time, the mass density of an accelerating object increases with time.

Since, $V(t)=\left[1-\beta(t)(u / c)^{2}\right]\left[1-(v / c)^{2}\right] \gamma(t) V_{o}$, we get the mass density $\rho(\mathrm{t})=\mathrm{m} /\left\{\left[1-\beta(\mathrm{t})(\mathrm{u} / \mathrm{c})^{2}\right]\left[1-(\mathrm{v} / \mathrm{c})^{2}\right] \gamma(\mathrm{t}) \mathrm{V}_{\mathrm{o}}\right\}$.

The accelerating object will turn itself into a transient black hole when $\rho(t)=0$. We know that $\rho(t)=0$ when, $\beta(t) u^{2}=c^{2}$, or $\mathrm{v}=\mathrm{c}$, or $\gamma(\mathrm{t})=0$.

Case- $1: \mathrm{v}=\mathrm{c}$

When $\lim _{v \rightarrow c} V(t)=0$, and $\lim _{V(t) \rightarrow 0} \rho(t)=\infty$.

Since $v=u+\alpha t$, when $v=c$, we have, $\mathrm{t}=(\mathrm{c}-\mathrm{u}) / \alpha$

In other words when time $t$ approaches $\mathrm{t}=(\mathrm{c}-\mathrm{u}) / \alpha, \alpha \neq 0$, the mass density of the accelerating object reaches infinity or bound less and turns itself into a black hole.

Case-2: $\beta(\mathrm{t}) \mathrm{u}^{2}=\mathrm{c}^{2}$

We know $\beta(t)$ is given by,

$$
\beta(\mathrm{t})=\left\{1+(\alpha \mathrm{t} / \mathrm{u})[2+\mathrm{H} / \mathrm{ct}]+(\alpha \mathrm{t} / \mathrm{u})^{2}\left[1+\mathrm{H} / \mathrm{ct}+\frac{1}{3}(\mathrm{H} / \mathrm{ct})^{2}\right]\right\}
$$

So, when $\beta(t) u^{2}=c^{2}$, we get,

$$
\mathrm{u}^{2}\left\{1+(\alpha \mathrm{t} / \mathrm{u})[2+\mathrm{H} / \mathrm{ct}]+(\alpha \mathrm{t} / \mathrm{u})^{2}\left[1+\mathrm{H} / \mathrm{ct}+\frac{1}{3}(\mathrm{H} / \mathrm{ct})^{2}\right]\right\}=\mathrm{c}^{2}
$$

Since $\mathrm{H} / \mathrm{ct}<<1$, we have, $\mathrm{u}^{2}+2 \alpha \mathrm{ut}+\alpha^{2} \mathrm{t}^{2}=\mathrm{c}^{2}$. That is,

$$
(\mathrm{u}+\alpha \mathrm{t})^{2}=\mathrm{c}^{2} \text {, or } \mathrm{t}=(-\mathrm{u} \pm \mathrm{c}) / \alpha .
$$

Since time $t$ has to be positive, $\mathrm{t}=(\mathrm{c}-\mathrm{u}) / \alpha$. When time $t$ 
approaches $\mathrm{t}=(\mathrm{c}-\mathrm{u}) / \alpha$, the volume $V(t)$ approaches zero and hence the mass density $\rho(t)$ approaches infinity turning itself into a transient black hole while the mass remains unchanged.

Case-3: $\gamma(\mathrm{t})=0$

We know $\gamma(t)$ is given by,

$$
\begin{gathered}
\gamma(\mathrm{t})=\varphi\left\{1-\frac{1}{2} \varphi\left[\alpha \mathrm{L}(\mathrm{c}+\mathrm{v}) /(\mathrm{c}-\mathrm{v}) \mathrm{c}^{2}\right]\right\} \\
\varphi=1+\alpha \mathrm{L} / \mathrm{c}(\mathrm{c}+\mathrm{v})
\end{gathered}
$$

where, $\mathrm{v}=\mathrm{u}+$ at. We know, $\gamma(\mathrm{t})=0$, when

$$
\begin{gathered}
1-\frac{1}{2} \varphi \alpha \mathrm{L}(\mathrm{c}+\mathrm{v}) /(\mathrm{c}-\mathrm{v}) \mathrm{c}^{2}=0 \\
2-\left(\alpha \mathrm{L} / \mathrm{c}^{2}\right)(\mathrm{c}+\mathrm{v}) /(\mathrm{c}-\mathrm{v})-(\alpha \mathrm{L} / \mathrm{c})^{2} / \mathrm{c}(\mathrm{c}-\mathrm{v})=0 \\
2 \mathrm{c}(\mathrm{c}-\mathrm{v})-(\alpha \mathrm{L} / \mathrm{c})(\mathrm{c}+\mathrm{v})-(\alpha \mathrm{L} / \mathrm{c})^{2}=0 \\
2 \mathrm{c}^{2}-2 \mathrm{cv}-\alpha \mathrm{L}-\alpha \mathrm{Lv} / \mathrm{c}-(\alpha \mathrm{L} / \mathrm{c})^{2}=0, \text { or } \\
(2 \mathrm{c}+\alpha \mathrm{L} / \mathrm{c}) \mathrm{v}=2 \mathrm{c}^{2}-\alpha \mathrm{L}-(\alpha \mathrm{L} / \mathrm{c})^{2} \\
\left(2+\alpha \mathrm{L} / \mathrm{c}^{2}\right) \mathrm{c} \mathrm{v}=\mathrm{c}^{2}\left[2-\alpha \mathrm{L} / \mathrm{c}^{2}-\left(\alpha \mathrm{L} / \mathrm{c}^{2}\right)^{2}\right] \\
\left(2+\alpha \mathrm{L} / \mathrm{c}^{2}\right) \mathrm{cv}=\mathrm{c}^{2}\left(2+\alpha \mathrm{L} / \mathrm{c}^{2}\right)\left(1-\alpha \mathrm{L} / \mathrm{c}^{2}\right), \text { that is } \\
\mathrm{v}=\mathrm{c}\left(1-\alpha \mathrm{L} / \mathrm{c}^{2}\right) .
\end{gathered}
$$

Since $\alpha \mathrm{L} / \mathrm{c}^{2}<<1$,

Using $\mathrm{v}=\mathrm{u}+\alpha \mathrm{t}$, we have,

$$
\mathrm{v}=\mathrm{c} .
$$

$$
\mathrm{u}+\alpha \mathrm{t}=\mathrm{c} \text {, }
$$$$
\mathrm{t}=(\mathrm{c}-\mathrm{u}) / \alpha \text {. }
$$

In all three cases, when the time $t$ approaches $\mathrm{t}=(\mathrm{c}-\mathrm{u}) / \alpha$, the volume of the accelerating object approaches zero, $V(t)=0$. Therefore the mass density of the accelerating object becomes unbounded or infinite; as time $t$ approaches $\mathrm{t}=(\mathrm{c}-\mathrm{u}) / \alpha, \lim _{V(t) \rightarrow 0} \rho(t)=\infty$,

As a result, an accelerating body turns itself into a black hole when time $t$ approaches $\mathrm{t}=(\mathrm{c}-\mathrm{u}) / \alpha$. The mass of the accelerating object remains unchanged. The same result can be obtained by using the Approximate General Volume Contraction given in Corollary-3.4.

Theorem-4.2: Mass Density Dilation

The mass density $\rho(t)$ of an accelerating object at time $t$ is given by, $\rho(\mathrm{t})=\rho_{\mathrm{o}} /\left[1-(\mathrm{v} / \mathrm{c})^{2}\right]^{2}$, where, $\mathrm{v}=\mathrm{u}+\alpha \mathrm{t}$, and $\rho_{o}$ is the mass density at stand still.

Proof: From Approximate General Volume Contraction Corollary-3.4, we have, $\mathrm{V}(\mathrm{t})=\left[1-(\mathrm{v} / \mathrm{c})^{2}\right]^{2} \mathrm{~V}_{\mathrm{o}}$, where $\mathrm{v}=\mathrm{u}+\alpha \mathrm{t}$.

If the mass of the object is $m$, since the mass is absolute, we get the mass density $\rho(t)$,

$$
\rho(\mathrm{t})=\mathrm{m} / \mathrm{V}(\mathrm{t})=\mathrm{m} /\left[1-(\mathrm{v} / \mathrm{c})^{2}\right]^{2} \mathrm{~V}_{\mathrm{o}}=\left(\mathrm{m} / \mathrm{V}_{\mathrm{o}}\right) /\left[1-(\mathrm{v} / \mathrm{c})^{2}\right]^{2}
$$

Since the mass density of the object at stand still, $\rho_{o}$ is given by, $\rho_{\mathrm{o}}=\mathrm{m} / \mathrm{V}_{\mathrm{o}}$. We have, $\rho(\mathrm{t})=\rho_{\mathrm{o}} /\left[1-(\mathrm{v} / \mathrm{c})^{2}\right]^{2}$, where, $\mathrm{v}=\mathrm{u}+\alpha \mathrm{t}$.

From this relationship also it is clear that the mass density become infinite when, $\mathrm{v}=\mathrm{c}$. Substituting for $v$ from $\mathrm{v}=\mathrm{u}+\mathrm{at}$, we have, $\mathrm{t}=(\mathrm{c}-\mathrm{u}) / \alpha$, In other words, an object becomes a transient black-hole when time approaches $\mathrm{t}=(\mathrm{c}-\mathrm{u}) / \alpha$.

\section{Gravity of an Accelerating Object}

The gravity dilation for an inertial frame is given in the Universal Relativity (URL) [5]. Here, we extend the gravity dilation to an accelerating frame.

Theorem-5.1: Surface Gravity Dilation

The surface gravity of an accelerating object dilates while the mass remains unchanged. The surface gravity dilation, $\Delta g$ given by, $\Delta \mathrm{g}=\varphi \mathrm{e}$, where, $\varphi$ is a constant in the range, $\mathrm{G} /(\mathrm{Rc})^{2} \leq \varphi \leq 2 \mathrm{G} /(\mathrm{Rc})^{2}, \mathrm{e}=\mathrm{mv}^{2}, G$ is the gravitational constant, $R$ is the radius of the spherical object at stand still, $c$ is the speed of light, speed of the object $\mathrm{v}=\mathrm{u}+\alpha \mathrm{t}, u$ is the inertial speed and $\alpha$ is the acceleration.

Proof: Let us consider a spherical object. At rest, the diameter is $2 R$ and the surface gravity $g=g_{o}$. When the object is accelerating the surface gravity will differ from point to point.

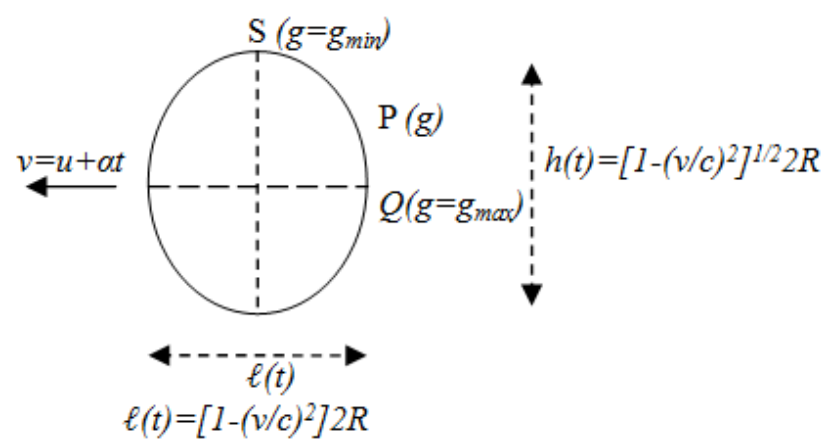

Figure 3. A Central Cross Section of an Accelerating Spherical Object (moving).

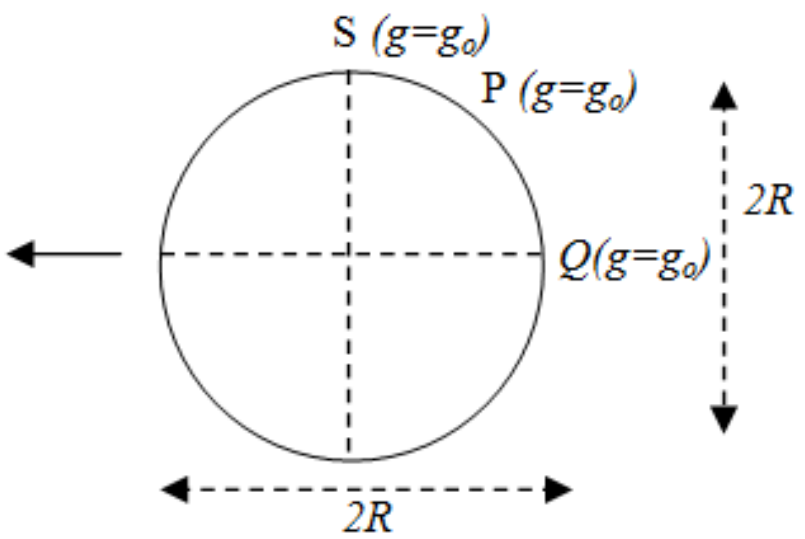

Figure 4. A Central Cross Section of the Stationary Spherical Object (at rest).

Though each point on the surface of the sphere has equal distances from the center $O$ when it is at rest, the moving object does not have an equal distance from the center to each point on the surface since the longitudinal contraction along the direction of the motion, and the lateral contraction perpendicular to the direction of motion are different. Consider a point $P$ on the surface at distance $r$ from the center of the moving object. The surface gravity at $P$ will be a minimum when $O P$ is perpendicular to the direction of motion; when $\mathrm{P}$ is at $\mathrm{S}$. The center and the center of the gravity of the sphere are the same from symmetry. 
Since the mass $m$ is absolute, the mass of the accelerating object is the same as the mass of the same object at rest. The surface gravity $g_{\min }$ of the moving object at point $S$, as well as the gravity $g_{o}$ when the object is at rest, are given by, $\mathrm{g}_{\min }=\mathrm{Gm} / \mathrm{r}^{2}(\mathrm{t}), \mathrm{g}_{\mathrm{o}}=\mathrm{Gm} / \mathrm{R}^{2}$, where $G$ is the gravitational constant, $m$ is the mass of the object, $r(t)$ is the distance to point $S$ on the surface from the center of gravity when the object is moving, $R$ is the distance to point $S$ on the surface from the center of gravity when the object is at rest; from the General Lateral Dimension Contraction Theorem-3.1 we know, $r(t) \leq R$. Using the gravitational relationships at $S$, we get, $\mathrm{g}_{\min } / \mathrm{g}_{\mathrm{o}}=[\mathrm{R} / \mathrm{r}(\mathrm{t})]^{2}$. From the Approximate General Lateral Contraction, Corollary-3.1, we have,

$\mathrm{r}(\mathrm{t})=\eta(\mathrm{t}) \mathrm{R}$, where, $\mathrm{n}(\mathrm{t})=\left[1-(\mathrm{v} / \mathrm{c})^{2}\right]^{1 / 2}, \mathrm{v}=\mathrm{u}+\alpha \mathrm{t}, u$ is the inertial speed, $\alpha$ is the acceleration. Substituting for $r(t)$, we have,

$\mathrm{g}_{\min }=\mathrm{g}_{\mathrm{o}} /\left[1-(\mathrm{v} / \mathrm{c})^{2}\right]$, where $\mathrm{v}=\mathrm{u}+\alpha \mathrm{t}$.

This indicates that the gravity of an accelerating object increases with time. When time approaches $\mathrm{t}=(\mathrm{c}-\mathrm{u}) / \alpha$, the speed of the object approaches the speed of light, and the gravity of the object reaches infinity while the mass remains unchanged. When $\mathrm{v}<<\mathrm{c}$, and $\mathrm{t}<<(\mathrm{c}-\mathrm{u}) / \alpha$, we have,

$$
\mathrm{g}_{\min } \mathrm{c}^{2}=\mathrm{g}_{\mathrm{o}} \mathrm{c}^{2}+\mathrm{g}_{\mathrm{o}} \mathrm{v}^{2} \text {, i.e., } \Delta \mathrm{g}_{\min } \mathrm{c}^{2}=\mathrm{g}_{\mathrm{o}} \mathrm{v}^{2} \text {, or, } \Delta \mathrm{g}_{\min }=\mathrm{g}_{\mathrm{o}}(\mathrm{v} / \mathrm{c})^{2},
$$

where $\Delta g_{\text {min }}=g_{-} g_{o}$. Since, $v=u+\alpha t$,

$$
\Delta \mathrm{g}_{\min }=\mathrm{g}_{\mathrm{o}}[(\mathrm{u}+\mathrm{at}) / \mathrm{c}]^{2} \text {. }
$$

Substituting for $g_{o}$, we get,

$\Delta g_{\text {min }}=\varphi_{\text {min }} e$,

where $\mathrm{e}=\mathrm{mv}^{2}$, the instantaneous kinetic energy of the moving object, $\varphi_{\min }=\mathrm{G} /(\mathrm{Rc})^{2}$, a constant, $\mathrm{v}=\mathrm{u}+\alpha \mathrm{t}$.

The kinetic energy of an accelerating object increases with time and hence the surface gravity of an accelerating object increases in time. It is the increase in surface gravity that the kinetic energy of an object is proportional to, not to the mass. By increasing the kinetic energy, we can increase the surface gravity of an object. Similarly, when the speed of an object deceases, the surface gravity decreases. The mass cannot be increased or decreased by changing its kinetic energy; mass is absolute.

In this relationship, the proportionality constant $\varphi$ varies according to the point $P$ chosen on the surface. If we chose a different point on the surface of the moving object, the distance from the center of gravity will be different and hence the proportionality constant $\varphi$ will be different. The surface gravity $g$ is the maximum when, $O P$, the line connecting the center of gravity $O$ and $P$ is on the direction of motion, that is when $P$ is on $Q$. We know that, $\mathrm{OQ}=\ell(\mathrm{t})$ and $\ell(\mathrm{t})=\left[1-(\mathrm{v} / \mathrm{c})^{2}\right] \mathrm{R}$, where $v$ is the instantaneous speed at time $t$ given by, $\mathrm{v}=\mathrm{u}+\alpha \mathrm{t}$, $u$ is the inertial speed, $\alpha$ is the acceleration. So, if the gravity at $\mathrm{Q}$ is $g_{\max }$, we get, $\mathrm{g}_{\max }=\mathrm{g}_{\mathrm{o}} /\left[1-(\mathrm{v} / \mathrm{c})^{2}\right]^{2}$. When speed $\mathrm{v}<<\mathrm{c}$, and time $\mathrm{t}<<(\mathrm{c}-\mathrm{u}) / \alpha$, we have, $\mathrm{g}_{\max }=\mathrm{g}_{\mathrm{o}}\left[1+2(\mathrm{v} / \mathrm{c})^{2}\right]$, and hence, $\Delta \mathrm{g}_{\max }=2 \mathrm{~g}_{\mathrm{o}}(\mathrm{v} / \mathrm{c})^{2}$, i.e.,

$$
\Delta \mathrm{g}_{\max }=\varphi_{\max } \mathrm{e},
$$

where $\varphi_{\max }=2 \mathrm{G} /(\mathrm{Rc})^{2}$, the instantaneous kinetic energy, $\mathrm{e}=\mathrm{mv}^{2}$.
So, we have the surface gravitation dilation, $\Delta g$ given by,

$$
\Delta \mathrm{g}=\varphi \mathrm{e},
$$

where $\mathrm{G} /(\mathrm{Rc})^{2} \leq \varphi \leq 2 \mathrm{G} /(\mathrm{Rc})^{2}, \mathrm{e}=\mathrm{mv}^{2}, \mathrm{v}=\mathrm{u}+\alpha \mathrm{t}$.

It is important to notice that $e$ is kinetic energy, not electromagnetic energy. It is not possible to substitute electromagnetic energy for $e$. They are two completely different forms of energy that are not equivalent although we can convert one to the other by different means.

When an object is moving, its mass remains constant, however its surface gravity increases due to the volume contraction. In the Special Relativity, this increase in gravity has been erroneously attributed to a mass dilation of a moving object that led to the misconceived relationship $\mathrm{e}=\mathrm{mc}^{2}$, which does not hold in reality since the time and mass are absolute. It is the surface gravity of a moving object that dilates, not the mass, and hence $\mathrm{e} \neq \mathrm{mc}^{2}$; there is no relativistic mass.

\section{The Relationship between Mass Density and Energy Density}

The mass is absolute. The mass does not depend on the observer's frame of reference; there is no such thing called relativistic mass. Since the mass is universal, it is not possible to change the mass of an object by changing the speed of the object. However, when an object is moving, volume of the object contracts and as a result the mass density dilates. When we consider a moving object, the energy is kinetic energy. It is not electromagnetic energy. It is not possible to use the kinetic energy in place of the electromagnetic energy or vice versa. The conversion of one form of energy to the other is a tedious process; ask an electrical engineer. There is no simple, elegant relationship between the electromagnetic energy and the kinetic energy, $e \neq m c^{2}$.

Theorem-6.1: The Mass Density and Energy Density Equivalence

The change of mass density $\Delta \rho(t)$ of an accelerating object is related to energy density by the relationship,

$$
\Delta \rho(\mathrm{t})=2 \rho_{\mathrm{o}}(\mathrm{v} / \mathrm{c})^{2},
$$

where $\Delta \rho(\mathrm{t})=\rho(\mathrm{t})-\rho_{\mathrm{o}}$ and $\mathrm{v}=\mathrm{u}+\alpha \mathrm{t}$.

Proof: From Mass Density Dilation Theorem-4.2, we have, $\rho(\mathrm{t})=\rho_{\mathrm{o}} /\left[1-(\mathrm{v} / \mathrm{c})^{2}\right]^{2}$, where, $\mathrm{v}=\mathrm{u}+\alpha \mathrm{t}$, and $\rho_{o}$ is the mass density at stand still. For $\mathrm{v}<<\mathrm{c}$ and $\mathrm{t}<<(\mathrm{c}-\mathrm{u}) / \alpha$, we have, $\rho(t)=\rho_{o}\left[1+2(v / c)^{2}\right]$, and hence,

$$
\begin{aligned}
& \Delta \rho(t)=2 \rho_{o}(v / c)^{2}, \text { i.e., } \\
& \Delta \rho(t)=2 \rho_{o}[(u+\alpha t) / c]^{2} .
\end{aligned}
$$

Compare this to the surface gravity dilation relationship, $\Delta \mathrm{g}_{\max }=2 \mathrm{~g}_{\mathrm{o}}(\mathrm{v} / \mathrm{c})^{2}$ given in the proof of Theorem-5.1.

As expected the mass density and kinetic energy density has the exactly the same form as the gravity and the kinetic energy density relationship; this is because the increase in gravity is the result of the increase in mass density. This equivalence indicates how kinetic energy density is translated into mass 
density. When the speed of an object increases, the mass density dilates. It is the mass density that dilates when an object is moving, not the mass. Similarly, when an object slows down, the mass density decreases. The mass always remains the same. It is not possible to increase the mass of an object by increasing its speed; the mass of an object cannot be reduced by decreasing the speed of the object either. The mass of an object is absolute; the mass of an object does not depend on the speed of an object or the frame of reference.

\section{The Universe}

Let us consider the information we have in front of us from observations. We are in the Milky Way galaxy. The nearby galaxies are moving away from us at a radial speed $\mathrm{u}_{\mathrm{i}}(\mathrm{t})=\partial\left[\mathrm{r}_{\mathrm{i}}(\mathrm{t})\right] / \partial \mathrm{t}$ proportional to the radial distance $\mathrm{r}_{\mathrm{i}}(\mathrm{t})$, $\forall \mathrm{i}=1,2, \ldots$, to the nearby galaxies from us. The nearby galaxies are moving away from $u$ at an acceleration $\alpha_{i}(t)=\partial\left[u_{i}(t)\right] / \partial t$ or $\alpha_{i}(t)=\partial^{2}\left[r_{i}(t)\right] / \partial t^{2}$.

One model that is used to explain the galactic red shift indicating that the galaxies are moving away from us is "the expanding universe" model. However, we can't say that the universe is expanding just because we see that the nearby galaxies are moving away from us. Can our proclamation that the universe is expanding because we saw that some distant objects are moving away from us be any different from the scenario where a cave-man who wondered out of the cave where he had been living all his life seen a distant moving object and proclaim that "the sky is falling"? It is hard to fathom how such an unrealistic expanding universe idea has taken hold to this day. The General Relativity $[2,10]$ had been used to justify the expanding universe model theoretically. However, the General Relativity does not hold true due to many factors [4] such as, the light is not relative, there is no space-time, time is not relative, and the principle of equivalence the General Relativity based on is incorrect. By seen an object in the sky that is moving away from us, it is totally unrealistic to suggest that the sky is expanding. What do you say if you see a galaxy, like Andromeda galaxy, coming towards you, "the sky is falling or contracting?" If we see many nearby galaxies are moving away while one galaxy is moving toward you, can you still say the sky is expanding? The sky contraction and the sky expansion can't take place at the same time. Just because we see few galaxies moving away from us, we can't say all the galaxies are moving away from us, and the sky is expanding. Without expansion or contraction of the space there will not be a big-bang either.

We need a realistic model rather than just to say "sky is falling" when we see a distant galaxy is moving toward us, or to say "sky is expanding" when a distant galaxy is moving away from us. Any realistic model should be able to explain individual behavior of each galaxy consistently under a single framework whether it is moving away from us, moving toward us, or at stand still. We need to find a realistic model from which we can derive theoretically the radial speed-distance relationship that we observe today when we look at distant galaxies.

\section{Galactic Cluster Model (GCM)}

It has been observed that the universe consists of Galactic Clusters [10]. In the Galactic Cluster Model (GCM) considered here, all the galaxies in the universe are groups into Local Galactic Clusters. The galaxies in a local cluster are orbiting around a one central galaxy in the cluster. In our Local Galactic Cluster, all the nearby galaxies are orbiting around the Milky Way galaxy. Several Local Galactic Clusters are orbiting around one Local Galactic Cluster to form a Local Super Galactic Cluster, and the pattern continues. The dynamics derived for one cluster can be equally applied to super clusters.

Let us consider our Local Galactic Cluster (LGC) centered on the Milky Way galaxy; we call it, the Milky Way Local Galactic Cluster (MW-LGC). Assume the state of the MW-LGC at time $t$ is: $M(t)=$ the mass of the Milky Way Galaxy, the orbiting center of our LGC; $m_{i}(t)=$ the mass of the $i^{\text {th }}$ orbiting galaxy; $r_{i}(t)=$ the distance to the $i^{\text {th }}$ galaxy from the Milky Way galaxy; $v_{i}(t)=$ the orbiting speed of $i^{\text {th }}$ galaxy

$\mathrm{u}_{\mathrm{i}}(\mathrm{t})=\frac{\partial}{\partial \mathrm{t}} \mathrm{r}_{\mathrm{i}}$, the radial speed of the $i^{\text {th }}$ galaxy,

$\frac{\partial}{\partial \mathrm{t}}\left[\mathrm{u}_{\mathrm{i}}\right]=$ radial acceleration of the $i^{\text {th }}$ galaxy, $\forall \mathrm{i}, \mathrm{i}=1,2, \ldots, \mathrm{n}-1$

where $n$ is the number of galaxies MW-LGC.

Now, we have galactic center, the Milky Way galaxy. We also have $n-1$ nearby galaxies orbiting around the center forming a one Local Galactic Cluster. We are located at the center of this LGC.

Theorem-8.1: Orbit Dilation (Galactic Radial Speed)

The radial speed, $\mathrm{u}_{\mathrm{i}}(\mathrm{t})$, that an orbiting galaxy moving away from the orbiting center of a Local Galactic Cluster (LGC) is given by $\mathrm{u}_{\mathrm{i}}(\mathrm{t})=\mathrm{H}_{\mathrm{i}}(\mathrm{t}) \mathrm{r}_{\mathrm{i}}(\mathrm{t})$, where,

$$
\mathrm{H}_{\mathrm{i}}(\mathrm{t})=\frac{\partial}{\partial t}\left\{\ln [\mathrm{M}(\mathrm{t})]+2 \ln \left[\mathrm{m}_{\mathrm{i}}(\mathrm{t})\right]\right\}, \forall \mathrm{i}, \mathrm{i}=1,2, \ldots, \mathrm{n}-1
$$

$r_{i}(t)$ is the orbit radius of the $i^{\text {th }}$ galaxy, $\ln$ is the logarithm. Proof: The state of a LGC at time $t$ is given by,

$$
\mathrm{S}\left[\mathrm{M}(\mathrm{t}), \mathrm{m}_{\mathrm{i}}(\mathrm{t}), \mathrm{v}_{\mathrm{i}}(\mathrm{t}), \mathrm{r}_{\mathrm{i}}(\mathrm{t}), \mathrm{u}_{\mathrm{i}}(\mathrm{t})\right] .
$$

The state of LGC at time $t+\Delta t$, where $\Delta \mathrm{t}$ is a small time interval, is given by,

$$
S\left[M(t+\Delta t), m_{i}(t+\Delta t), v_{i}(t+\Delta t), r_{i}(t+\Delta t), u_{i}(t+\Delta t)\right] .
$$

Now, we have,

$$
\begin{gathered}
r_{i}(t+\Delta t)=r_{i}(t)+\Delta r_{i}(t) \\
v_{i}(t+\Delta t)=v_{i}(t)+\Delta v_{i}(t) \\
m_{i}(t+\Delta t)=m_{i}(t)+\Delta m_{i}(t) \\
M(t+\Delta t)=M(t)+\Delta M(t)
\end{gathered}
$$

By using the gravitational mechanics, we have,

$$
\begin{gathered}
{[\mathrm{M}(\mathrm{t}+\Delta \mathrm{t}) / \mathrm{M}(\mathrm{t})]=\left[\mathrm{v}_{\mathrm{i}}(\mathrm{t}+\Delta \mathrm{t}) / \mathrm{v}_{\mathrm{i}}(\mathrm{t})\right]^{2}\left[\mathrm{r}_{\mathrm{i}}(\mathrm{t}+\Delta \mathrm{t}) / \mathrm{r}_{\mathrm{i}}(\mathrm{t})\right]} \\
{\left[\mathrm{v}_{\mathrm{i}}(\mathrm{t}+\Delta \mathrm{t}) / \mathrm{v}_{\mathrm{i}}(\mathrm{t})\right]=\left[\mathrm{m}_{\mathrm{i}}(\mathrm{t}) / \mathrm{m}_{\mathrm{i}}(\mathrm{t}+\Delta \mathrm{t})\right]}
\end{gathered}
$$

From eqns. (6) and (7) we get, 


$$
\begin{aligned}
& {[\mathrm{M}(\mathrm{t}+\Delta \mathrm{t}) / \mathrm{M}(\mathrm{t})]=\left[\mathrm{m}_{\mathrm{i}}(\mathrm{t}) / \mathrm{m}_{\mathrm{i}}(\mathrm{t}+\Delta \mathrm{t})\right]^{2}\left[\mathrm{r}_{\mathrm{i}}(\mathrm{t}+\Delta \mathrm{t}) / \mathrm{r}_{\mathrm{i}}(\mathrm{t})\right]} \\
& \mathrm{r}_{\mathrm{i}}(\mathrm{t}+\Delta \mathrm{t})=[\mathrm{M}(\mathrm{t}+\Delta \mathrm{t}) / \mathrm{M}(\mathrm{t})]\left[\mathrm{m}_{\mathrm{i}}(\mathrm{t}+\Delta \mathrm{t}) / \mathrm{m}_{\mathrm{i}}(\mathrm{t})\right]^{2} \mathrm{r}_{\mathrm{i}}(\mathrm{t})
\end{aligned}
$$

Using eqns. (4) and (5) in eqn. (8), we get,

$$
r_{i}(t+\Delta t)=[1+\Delta M(t) / M(t)]\left[1+\Delta m_{i}(t) / m_{i}(t)\right]^{2} r_{i}(t)
$$

Since $\Delta \mathrm{M}(\mathrm{t}) / \mathrm{M}(\mathrm{t})<<1$ and $\Delta \mathrm{m}_{\mathrm{i}}(\mathrm{t}) / \mathrm{m}_{\mathrm{i}}(\mathrm{t})<<1$, we have,

$$
r_{i}(t+\Delta t)=\left[1+\Delta M(t) / M(t)+2 \Delta m_{i}(t) / m_{i}(t)\right] r_{i}(t)
$$

From eqns. (2), and (10), we get,

$$
\Delta \mathrm{r}_{\mathrm{i}}(\mathrm{t})=\left[\Delta \mathrm{M}(\mathrm{t}) / \mathrm{M}(\mathrm{t})+2 \Delta \mathrm{m}_{\mathrm{i}}(\mathrm{t}) / \mathrm{m}_{\mathrm{i}}(\mathrm{t})\right] \mathrm{r}_{\mathrm{i}}(\mathrm{t})
$$

By dividing $\Delta t$, we get,

$$
\left[\Delta \mathrm{r}_{\mathrm{i}}(\mathrm{t}) / \Delta \mathrm{t}\right]=\frac{1}{M(t)}[\Delta \mathrm{M}(\mathrm{t}) / \Delta \mathrm{t}] \mathrm{r}_{\mathrm{i}}(\mathrm{t})+\left(2 / \mathrm{m}_{\mathrm{i}}(\mathrm{t})\right)\left[\Delta \mathrm{m}_{\mathrm{i}}(\mathrm{t}) / \Delta \mathrm{t}\right] \mathrm{r}_{\mathrm{i}}(\mathrm{t})
$$

By taking the limit $\Delta \mathrm{t} \rightarrow 0$, we get,

$$
\begin{gathered}
\lim _{\Delta \mathrm{t} \rightarrow 0} \Delta \mathrm{r}_{\mathrm{i}}(\mathrm{t}) / \Delta \mathrm{t}=\frac{\partial}{\partial t}\left[\mathrm{r}_{\mathrm{i}}(\mathrm{t})\right], \lim _{\Delta \mathrm{t} \rightarrow 0} \Delta \mathrm{M}(\mathrm{t}) / \Delta \mathrm{t}=\frac{\partial}{\partial t}[\mathrm{M}(\mathrm{t})] \\
\lim _{\Delta \mathrm{t} \rightarrow 0} \Delta \mathrm{m}_{\mathrm{i}}(\mathrm{t}) / \Delta \mathrm{t}=\frac{\partial}{\partial t}\left[\mathrm{~m}_{\mathrm{i}}(\mathrm{t})\right] . \text { From eqn. (12), we get, } \\
\frac{\partial}{\partial t}\left[\mathrm{r}_{\mathrm{i}}(\mathrm{t})\right]=\left\{\left[\frac{1}{M(t)} \frac{\partial}{\partial t} \mathrm{M}(\mathrm{t})\right]+\left[\left(2 / \mathrm{m}_{\mathrm{i}}(\mathrm{t})\right) \frac{\partial}{\partial t} \mathrm{~m}_{\mathrm{i}}(\mathrm{t})\right]\right\} \mathrm{r}_{\mathrm{i}}(\mathrm{t})
\end{gathered}
$$

Since $u_{i}(t)=\frac{\partial}{\partial t} r_{i}(t)$, we get, $\mathrm{u}_{\mathrm{i}}(\mathrm{t})=\left\{\frac{\partial}{\partial \mathrm{t}}\left\{\ln [\mathrm{M}(\mathrm{t})]+2 \ln \left[\mathrm{m}_{\mathrm{i}}(\mathrm{t})\right]\right\}\right\} \mathrm{r}_{\mathrm{i}}(\mathrm{t})$,

where $\ln (\mathrm{)}$ is the natural logarithm.

Let, $\mathrm{H}_{\mathrm{i}}(\mathrm{t})=\frac{\partial}{\partial \mathrm{t}}\left\{\ln [\mathrm{M}(\mathrm{t})]+2 \ln \left[\mathrm{m}_{\mathrm{i}}(\mathrm{t})\right]\right\}$, then, we have,

$\mathrm{u}_{\mathrm{i}}(\mathrm{t})=\mathrm{H}_{\mathrm{i}}(\mathrm{t}) \mathrm{r}_{\mathrm{i}}(\mathrm{t}), \forall \mathrm{i}=1,2, \ldots \mathrm{n}-1 . \mathrm{H}_{\mathrm{i}}(\mathrm{t})$ is the orbit dilation parameter of $i^{\text {th }}$ galaxy with mass $m_{i}$ and orbiting center mass $M$. The galactic orbit dilation is proportional to the derivative of the galactic mass.

In the case of our Local Galactic Cluster, $M$ is the mass of the Milky Way galaxy, $m_{i}$ is the $i^{\text {th }}$ galaxy in the Milky Way Local Galactic Cluster (MW-LGC). The change of mass of the Local Galactic Center, the Milky Way galaxy, as well as the change in the mass of a galaxy in the cluster contributes to the radial speed that galaxy moving away from us. The further the galaxy away from us, the faster it is moving away from us. This is exactly the relationship that has been observed of the nearby galaxies $[8,10]$. We are at the orbiting center of a Local Galactic Cluster (LGC).

Property:

If the diameter of a Local Galactic Cluster is $R$, then

$\mathrm{R}<\mathrm{c} / \mathrm{H}_{\max }$, where $\mathrm{H}_{\max }$ is the largest orbit dilation parameter $\mathrm{H}_{\mathrm{i}}, \forall \mathrm{i}=1,2,3, \ldots \mathrm{n}-1$.

This property prevents the radial speed of any galaxy ever exceeding the speed of light, i.e. $\mathbf{u}<\mathbf{c}$. All the galaxies within any cluster are within the radius $\mathrm{c} / \mathrm{H}_{\max }$. Any galaxy outside this radius will be a member of a different Local Galactic Cluster. Since $H$ is not a constant, there is no unbounded radial speed of galaxies; the value of $H$ is determined by the rate of change of mass of galaxies.

Newton thought some sort of, once in a while, unknown external intervention (Newton called it divine intervention; there is no such thing called divine) is necessary in keeping the orbiting bodies from drifting away from their orbits. However, no such external intervention is required, and no such external authority exists. Whether it is a planet, planetary system, galaxy, or Local Galactic Cluster, as the mass of the orbiting object changes over time, their orbits get adjusted on their own automatically based on the rate of change of mass. The orbits are dilated or contracted according to the rate of change of mass; orbiting objects are auto-tuned. We can see the auto-tuning in action from the observation that some galaxies are moving away while the others are moving toward us. The mass of any object in the universe is time varying, and as a result the orbit of any object in the universe will be time varying. The change of mass of a planet is so subtle that we do not notice any change in its orbit unless it comes into collision with an asteroid or another object. Such a collision creates an abrupt change of mass of a planet resulting in dilation of the planet's orbit, and hence sudden cooling, bringing with it the ice-age; the ice-age similar to the one that made the dinosaurs extinct.

Theorem-8.2: Orbit Acceleration (Galactic Radial Acceleration)

The radial acceleration, $\frac{\partial}{\partial t} u_{i}(t)$, that an orbiting galaxy moving away from the orbiting center is given by

$$
\begin{gathered}
\frac{\partial}{\partial t} \mathrm{u}_{\mathrm{i}}(\mathrm{t})=\Phi_{\mathrm{i}}(\mathrm{t}) \mathrm{r}_{\mathrm{i}}(\mathrm{t}), \text { where, } \\
\Phi_{\mathrm{i}}(\mathrm{t})=\mathrm{H}_{\mathrm{i}}^{2}(\mathrm{t})+\frac{\partial}{\partial t} \mathrm{H}_{\mathrm{i}}(\mathrm{t}), \\
\mathrm{H}_{\mathrm{i}}(\mathrm{t})=\frac{\partial}{\partial t}\left\{\ln [\mathrm{M}(\mathrm{t})]+2 \ln \left[\mathrm{m}_{\mathrm{i}}(\mathrm{t})\right]\right\}, \quad \forall \mathrm{i}, \mathrm{i}=1,2, \ldots, \mathrm{n} .
\end{gathered}
$$

$r_{i}(t)$ is the orbit radius of the $i^{\text {th }}$ galaxy.

Proof: From Orbit Dilation Theorem-8.1, we have,

$\mathrm{u}_{\mathrm{i}}(\mathrm{t})=\mathrm{H}_{\mathrm{i}}(\mathrm{t}) \mathrm{r}_{\mathrm{i}}(\mathrm{t}), \forall \mathrm{i}=1,2, \ldots \mathrm{n}-1$. Differentiating with respect to time $t$, we get,

$$
\begin{gathered}
\frac{\partial}{\partial t} \mathrm{u}_{\mathrm{i}}(\mathrm{t})=\mathrm{H}_{\mathrm{i}}(\mathrm{t}) \frac{\partial}{\partial t} \mathrm{r}_{\mathrm{i}}(\mathrm{t})+\mathrm{r}_{\mathrm{i}}(\mathrm{t}) \frac{\partial}{\partial t} \mathrm{H}_{\mathrm{i}}(\mathrm{t}) \\
=\mathrm{H}_{\mathrm{i}}(\mathrm{t}) \mathrm{u}_{\mathrm{i}}(\mathrm{t})+\mathrm{r}_{\mathrm{i}}(\mathrm{t}) \frac{\partial}{\partial t} \mathrm{H}_{\mathrm{i}}(\mathrm{t})
\end{gathered}
$$

Substituting for $\mathrm{u}_{\mathrm{i}}(\mathrm{t})$, we get,

$$
\frac{\partial}{\partial \mathrm{t}} \mathrm{u}_{\mathrm{i}}(\mathrm{t})=\Phi_{\mathrm{i}}(\mathrm{t}) \mathrm{r}_{\mathrm{i}}(\mathrm{t})
$$

where $\Phi_{\mathrm{i}}(\mathrm{t})$ is the orbit acceleration parameter given by,

$$
\Phi_{\mathrm{i}}(\mathrm{t})=\mathrm{H}_{\mathrm{i}}^{2}(\mathrm{t})+\frac{\partial}{\partial t} \mathrm{H}_{\mathrm{i}}(\mathrm{t})
$$

$\mathrm{H}_{\mathrm{i}}(\mathrm{t})$ is the orbit dilation parameter of the $i^{\text {th }}$ galaxy with mass $m_{i}(t)$ with orbiting center galaxy of mass $M(t)$.

Theorem-8.3: Approximate Orbit Acceleration (Galactic Radial Acceleration)

The approximate radial acceleration, $\frac{\partial}{\partial \mathrm{t}} \mathrm{u}_{\mathrm{i}}(\mathrm{t})$, that an orbiting galaxy moving away from the orbiting center is given by, $\frac{\partial}{\partial \mathrm{t}} \mathrm{u}_{\mathrm{i}}(\mathrm{t})=\Phi_{\mathrm{i}}(\mathrm{t}) \mathrm{r}_{\mathrm{i}}(\mathrm{t})$, where,

$$
\Phi_{\mathrm{i}}(\mathrm{t}) \approx(1 / \mathrm{M}(\mathrm{t})) \frac{\partial}{\partial t} \frac{\partial}{\partial t}[\mathrm{M}(\mathrm{t})]+(2 / \mathrm{m}(\mathrm{t})) \frac{\partial}{\partial t} \frac{\partial}{\partial t}\left[\mathrm{~m}_{\mathrm{i}}(\mathrm{t})\right],
$$

$\forall \mathrm{i}, \mathrm{i}=1,2, \ldots, \mathrm{n}-1, \mathrm{r}_{\mathrm{i}}(\mathrm{t})$ is the orbit radius of the $i^{\text {th }}$ galaxy. 
Proof: From the Orbit Acceleration Theorem-8.2, we have,

$$
\begin{aligned}
& \frac{\partial}{\partial t} \mathrm{u}_{\mathrm{i}}(\mathrm{t})=\Phi_{\mathrm{i}}(\mathrm{t}) \mathrm{r}_{\mathrm{i}}(\mathrm{t}), \text { where, } \Phi_{\mathrm{i}}(\mathrm{t})=\mathrm{H}_{\mathrm{i}}^{2}(\mathrm{t})+\frac{\partial}{\partial t} \mathrm{H}_{\mathrm{i}}(\mathrm{t}), \\
& \mathrm{H}_{\mathrm{i}}(\mathrm{t})=\frac{\partial}{\partial t}\left\{\ln [\mathrm{M}(\mathrm{t})]+2 \ln \left[\mathrm{m}_{\mathrm{i}}(\mathrm{t})\right]\right\}, \forall \mathrm{i}, \mathrm{i}=1,2, \ldots, \mathrm{n} .
\end{aligned}
$$

$r_{i}(t)$ is the orbit radius of the $i^{\text {th }}$ galaxy.

$$
\begin{aligned}
& \mathrm{H}_{\mathrm{i}}^{2}(\mathrm{t})=\left\{\left[(1 / \mathrm{M}(\mathrm{t})) \frac{\partial}{\partial t} \mathrm{M}(\mathrm{t})\right]+\left[\left(2 / \mathrm{m}_{\mathrm{i}}(\mathrm{t})\right) \frac{\partial}{\partial t} \mathrm{~m}_{\mathrm{i}}(\mathrm{t})\right]\right\}^{2} . \\
&=\left[(1 / \mathrm{M}(\mathrm{t})) \frac{\partial}{\partial t} \mathrm{M}(\mathrm{t})\right]^{2}+ \\
&\left(4 / \mathrm{M}(\mathrm{t}) \mathrm{m}_{\mathrm{i}}(\mathrm{t})\right)\left[\frac{\partial}{\partial t} \mathrm{M}(\mathrm{t})\right]\left[\frac{\partial}{\partial t} \mathrm{~m}_{\mathrm{i}}(\mathrm{t})\right]+\left[\left(2 / \mathrm{m}_{\mathrm{i}}(\mathrm{t})\right) \frac{\partial}{\partial t} \mathrm{~m}_{\mathrm{i}}(\mathrm{t})\right]^{2} . \\
& \frac{\partial}{\partial t} \mathrm{H}_{\mathrm{i}}(\mathrm{t})=\frac{\partial}{\partial t}\left\{\left[(1 / \mathrm{M}(\mathrm{t})) \frac{\partial}{\partial t} \mathrm{M}(\mathrm{t})\right]+\left[\left(2 / \mathrm{m}_{\mathrm{i}}(\mathrm{t})\right) \frac{\partial}{\partial t} \mathrm{~m}_{\mathrm{i}}(\mathrm{t})\right]\right\} \\
&=\left[(1 / \mathrm{M}(\mathrm{t})) \frac{\partial}{\partial t} \frac{\partial}{\partial t} \mathrm{M}(\mathrm{t})\right]-\left[\frac{\partial}{\partial t} \ln \mathrm{M}(\mathrm{t})\right]^{2} \\
&+\left[\left(2 / \mathrm{m}_{\mathrm{i}}(\mathrm{t})\right) \frac{\partial}{\partial t} \frac{\partial}{\partial t} \mathrm{~m}_{\mathrm{i}}(\mathrm{t})\right]-2\left[\frac{\partial}{\partial t} \ln \mathrm{m}_{\mathrm{i}}(\mathrm{t})\right]^{2} .
\end{aligned}
$$

Since $M$ is the mass of the central galaxy that exert a gravitational influence on the rest of the orbiting galaxies,

$\mathrm{M} \gg>\mathrm{m}_{\mathrm{i}}, \forall \mathrm{i}, \mathrm{i}=1,2, \ldots, \mathrm{n}-1$. Therefore, we have,

$$
\begin{gathered}
\mathrm{H}_{\mathrm{i}}{ }^{2}(\mathrm{t})<<\frac{\partial}{\partial t} \mathrm{H}_{\mathrm{i}}(\mathrm{t}) \text {, and } \\
\Phi_{\mathrm{i}}(\mathrm{t})=(1 / \mathrm{M}(\mathrm{t})) \frac{\partial}{\partial t} \frac{\partial}{\partial t} \mathrm{M}(\mathrm{t})-\left[\frac{\partial}{\partial t} \ln \mathrm{M}(\mathrm{t})\right]^{2} \\
+\left(2 / \mathrm{m}_{\mathrm{i}}(\mathrm{t})\right) \frac{\partial}{\partial t} \frac{\partial}{\partial t} \mathrm{~m}_{\mathrm{i}}(\mathrm{t})-2\left[\frac{\partial}{\partial t} \ln \mathrm{m}_{\mathrm{i}}(\mathrm{t})\right]^{2} . \\
\text { Since }(1 / \mathrm{M}(\mathrm{t})) \frac{\partial}{\partial t} \frac{\partial}{\partial t} \mathrm{M}(\mathrm{t})>>\left[\frac{\partial}{\partial t} \ln \mathrm{M}(\mathrm{t})\right]^{2}
\end{gathered}
$$

and $\left(2 / \mathrm{m}_{\mathrm{i}}(\mathrm{t})\right)\left\{\frac{\partial}{\partial \mathrm{t}} \frac{\partial}{\partial \mathrm{t}}\left[\mathrm{m}_{\mathrm{i}}(\mathrm{t})\right]>>2\left\{\frac{\partial}{\partial \mathrm{t}} \ln \left[\mathrm{m}_{\mathrm{i}}(\mathrm{t})\right]\right\}^{2}\right.$ we have, $\Phi_{\mathrm{i}}(\mathrm{t}) \cong(1 / \mathrm{M}(\mathrm{t})) \frac{\partial}{\partial \mathrm{t}} \frac{\partial}{\partial \mathrm{t}}[\mathrm{M}(\mathrm{t})]+\left(2 / \mathrm{m}_{\mathrm{i}}(\mathrm{t})\right) \frac{\partial}{\partial \mathrm{t}} \frac{\partial}{\partial \mathrm{t}}\left[\mathrm{m}_{\mathrm{i}}(\mathrm{t})\right]$

where, $M(t)$ is the orbiting center mass of the Local Galactic Cluster (LGC), and $m_{i}(t)$ is the mass of the $i^{\text {th }}$ galaxy in the cluster at time $t$. The galactic orbit acceleration is proportional to the second derivative of the galactic masses.

Theorem-8.4: Realistic Approximate Orbit Dilation [7]

The radial speed of the $i^{\text {th }}$ galaxy of mass $m_{\mathrm{i}}$ in a Local Galactic Cluster (LGC) with a orbiting center mass $M$, where $\mathrm{M}>>m_{i}$, is given by,

$\mathrm{u}_{\mathrm{i}}(\mathrm{t})=\mathrm{H}_{\mathrm{i}}(\mathrm{t}) \mathrm{r}_{\mathrm{i}}(\mathrm{t})$, where,

$\mathrm{H}_{\mathrm{i}}(\mathrm{t})=2 \frac{\partial}{\partial t} \ln \left[\mathrm{m}_{\mathrm{i}}(\mathrm{t})\right], \forall \mathrm{i}, \mathrm{i}=1,2, \ldots, \mathrm{n}-1$,

$r_{i}(t)$ is the orbit radius of the $i^{\text {th }}$ galaxy.

Proof: In a Local Galactic Cluster, the galaxy at the center exerts a gravitation influence on the rest of the galaxies. The rest of the galaxies in the Local Galactic Cluster are orbiting around the orbiting center, which is a galaxy at the center.

In order for the galaxy at the center of the Local Galactic Cluster to have a gravitational influence on the rest of the galaxies in the Local Galactic Cluster (LGC), the mass of the galaxy at the center of the galactic cluster where the rest of the galaxies are orbiting around should be significantly mush higher than the rest of the galaxies in the galactic cluster. Therefore, we have, $\mathrm{M}>>\mathrm{m}_{\mathrm{i}}(\mathrm{t}), \forall \mathrm{i}, \mathrm{i}=1,2, \ldots, \mathrm{n}-1$.

From the orbit dilation Theorem-8.1, we have, $\mathrm{u}_{\mathrm{i}}(\mathrm{t})=\mathrm{H}_{\mathrm{i}}(\mathrm{t}) \mathrm{r}_{\mathrm{i}}(\mathrm{t})$ where, $\mathrm{H}_{\mathrm{i}}(\mathrm{t})=\frac{\partial}{\partial \mathrm{t}}\left\{\ln [\mathrm{M}(\mathrm{t})]+2 \ln \left[\mathrm{m}_{\mathrm{i}}(\mathrm{t})\right]\right\}, \forall \mathrm{i}, \mathrm{i}=1,2, \ldots, \mathrm{n}-1$ $r_{i}(t)$ is the orbit radius of the $i^{\text {th }}$ galaxy.

$$
\mathrm{H}_{\mathrm{i}}(\mathrm{t})=(1 / \mathrm{M}(\mathrm{t})) \frac{\partial}{\partial \mathrm{t}}[\mathrm{M}(\mathrm{t})]+\left(2 / \mathrm{m}_{\mathrm{i}}(\mathrm{t})\right) \frac{\partial}{\partial \mathrm{t}}\left[\mathrm{m}_{\mathrm{i}}(\mathrm{t})\right] \text {, }
$$

Since $1 / M(t)<<1 / m_{i}(t)$, we have,

$$
\begin{gathered}
\mathrm{H}_{\mathrm{i}}(\mathrm{t}) \cong\left(2 / \mathrm{m}_{\mathrm{i}}(\mathrm{t})\right) \frac{\partial}{\partial \mathrm{t}}\left[\mathrm{m}_{\mathrm{i}}(\mathrm{t})\right], \\
\mathrm{H}_{\mathrm{i}}(\mathrm{t})=2 \frac{\partial}{\partial t} \ln \left[\mathrm{m}_{\mathrm{i}}(\mathrm{t})\right], \forall \mathrm{i}, \mathrm{i}=1,2, \ldots, \mathrm{n}-1 .
\end{gathered}
$$

Theorem-8.5: Realistic Approximate Orbit Acceleration [7] The radial acceleration of the $i^{\text {th }}$ galaxy of mass $m_{\mathrm{i}}$ in a Local Galactic Cluster (LGC) with a orbiting center mass $M$, where $\mathrm{M}>>\mathrm{m}_{\mathrm{i}}$, is given by,

$$
\begin{gathered}
\frac{\partial}{\partial t} \mathrm{u}_{\mathrm{i}}(\mathrm{t})=\Phi_{\mathrm{i}}(\mathrm{t}) \mathrm{r}_{\mathrm{i}}(\mathrm{t}), \text { where, } \\
\Phi_{\mathrm{i}}(\mathrm{t}) \cong(2 / \mathrm{m}(\mathrm{t})) \frac{\partial}{\partial t} \frac{\partial}{\partial t} \mathrm{~m}_{\mathrm{i}}(\mathrm{t}),
\end{gathered}
$$

$\forall \mathrm{i}, \mathrm{i}=1,2, \ldots, \mathrm{n}-1, r_{i}(t)$ is the orbit radius of the $i^{\text {th }}$ galaxy.

Proof: For a Local Galactic cluster to exist, the mass of the galactic center must be significantly much higher than the rest of the galaxies in the Local Galactic Cluster (LGC) and hence, $\mathrm{M}>>\mathrm{m}_{\mathrm{i}}$. From the Approximate Orbit Acceleration Theorem-8.2, we have,

$$
\begin{gathered}
\frac{\partial}{\partial t} \mathrm{u}_{\mathrm{i}}(\mathrm{t})=\Phi_{\mathrm{i}}(\mathrm{t}) \mathrm{r}_{\mathrm{i}}(\mathrm{t}), \text { where, } \\
\Phi_{\mathrm{i}}(\mathrm{t}) \cong(1 / \mathrm{M}(\mathrm{t})) \frac{\partial}{\partial t} \frac{\partial}{\partial t}[\mathrm{M}(\mathrm{t})]+(2 / \mathrm{m}(\mathrm{t})) \frac{\partial}{\partial t} \frac{\partial}{\partial t}\left[\mathrm{~m}_{\mathrm{i}}(\mathrm{t})\right],
\end{gathered}
$$

$\forall \mathrm{i}, \mathrm{i}=1,2, \ldots, \mathrm{n}-1, r_{i}(t)$ is the orbit radius of the $i^{\text {th }}$ galaxy. Since $M(t)>>m_{i}(t)$, we have

$$
\Phi_{\mathrm{i}}(\mathrm{t}) \cong(2 / \mathrm{m}(\mathrm{t})) \frac{\partial}{\partial t} \frac{\partial}{\partial t} \mathrm{~m}_{\mathrm{i}}(\mathrm{t}), \forall \mathrm{i}, \mathrm{i}=1,2, \ldots, \mathrm{n}-1
$$

\section{Universe in a New Light}

Here, we are going to consider answers to several common questions based on our new found understanding. Let us recap what we know so far $[4,5,6,7]$ :

a). The light is not relative. The light has no mass.

b). Time is absolute, does not depend on the frame of reference.

c). Mass is absolute, does not depend on the frame of reference; there is no such thing called "relative-mass".

d). Time does not depend on space; there is no space-time.

e). There is neither a beginning of time nor an end of time.

f). There is no fourth dimension; time is a definition, not a dimension.

g). You can't create a fourth space dimension-ct by multiplying time $t$ by $c$, the speed of light; it only exists in your notebook, not in space.

h). Every "things" are discrete; they have mass; movement of anything that has a mass is relative.

i). Every "non-things" are continuous; they have no mass; their movements are not relative; "non-things" are not the same as "no-things".

j). Time is continuous, because it is not a "thing". 
k). Space is continuous, because it is not a "thing".

1). Space does exist; it is not a definition; so we can travel in space.

$\mathrm{m})$. Time does not exist because time is a definition; so we can't travel in time.

n). You can travel in 3-dimensional space either forward or backward; it is always you that travel, never the space.

o). You can travel in time neither forward nor backward; it is always the time that travels, not you; you just enjoy, suffer, or bear the ride; you have no other choice; you can't stop time.

p). A moving body contracts in all directions since the speed of light depends only on the permittivity and the permeability of the medium, and nothing else.

q). Surface gravity of a moving body dilates.

r). Masses of galaxies and planets are time varying, not constants; the mass of any object is not a constant.

s). Orbits of planets, stars, galaxies, Local Galactic Clusters (LGC), Super Local Galactic Clusters (SLGC)... are time varying, not fixed; orbits are self adjusting or auto-tuned according to rate of change of mass.

t). It is the rate of change of mass of an object and the rate of change of mass of the orbital center that causes the orbit of an object to dilate or contract.

u). Galactic orbits contract, dilate or remains steady.

v). Planetary orbits usually undergo orbit contraction due to mass loss, however, in the event of a collision with an asteroid or a meteorite, planetary orbits dilate.

w). A collision of earth with an asteroid will bring the earth into a sudden ice age due to abrupt orbit dilation, making the planet un-inhabitable just as it did to the species in the era of dinosaurs.

$\mathrm{x})$. Universe is not expanding.

y). The idea of expanding universe is a result of "cave-man's syndrome".

z). Special relativity is incorrect (correct approach, but wrong conclusions).

aa). General relativity is incorrect (wrong approach, and wrong conclusions).

ab). There are no worm holes or pot holes or any other holes in space; they only exist in "things"; space is a "non-thing".

ac). There is/was no big-bang.

ad). Snow on an off-tuned television is thermal noise, not some remnant from a hypothetical big-bang (a big-nonsense).

ae). Only a "thing" that has a beginning, ages.

af). Any "thing" that has a beginning must have an end.

ag). Universe has no beginning and hence it has no age; it goes through a change and we use that change to define time; the claim that "the universe is 13.5 billion years old" is simply groundless since the universe has no beginning.

ah). $e \neq m c^{2}$ and why everybody should know about it (a bad book title).

ai). Light is always a wave, never a particle.

aj). There are no random particles of light or photons; what we have are bursts of lights.

ak). If the light consists of random particles known as photons, there can never be directional and coherent light rays, because photons by definition have a random location in space.

al). Particles are not waves and waves are not particles.

am). Kinetic energy of a particle is not electromagnetic energy.

an). Kinetic energy $\neq h f_{n}$; only electromagnetic energy $=h f_{n}$.

ao). Spectrum of light is discrete, $f_{n}=n e / h, \forall n=1,2, \ldots$ where $e$ is quantum energy and $h$ is the Plank constant.

ap). Frequency resolution of light is $e / h$.

aq). Light consists of wave bursts of duration $F=h / e$, a universal constant.

ar). Once a burst of light wave is out of a source, it has nothing to do with the source anymore; it travels according to Maxwell's equations.

as). A path light burst takes is completely determined by the electrical properties of the medium, nothing else.

at). Accelerating body turns itself into a black hole as time approaches $t=(c-u) / \alpha, \alpha$ is the acceleration, $u$ is the inertial speed, and $c$ is the speed of light; principle of equivalence does not hold true.

au). Universe consists of orbiting Galactic Clusters.

av). Each galactic cluster has an orbiting center where the rest of the galaxies in the cluster are orbiting.

aw). We are in the Milky Way Local Galactic Cluster (MW-LGC) where the nearby galaxies in the cluster are orbiting around the Milky Way Galaxy.

ax). The dilation of a galactic orbit is proportional to the rate of change of its mass, and to the radial distance from the orbiting center.

ay). Since orbit dilation is proportional to the radial distance, orbit dilation always takes place at an accelerated rate.

az). Speed is the currency for any object to be independent in the space (money in the personal bank account).

ba). Each object in the space has to be moving in its own orbit to be independent.

bb). If the earth is hit by an asteroid, the rate of change of mass of the earth is so significant since the hit is so sudden; it will result in an abrupt dilation of the earth's orbit bringing in the ice-age that led to the extinction of species in the era of dinosaurs.

bc). Abrupt orbit dilation will create our own demise as well as the demise many other species in our time just as it did for dinosaurs.

bd). There may be blue planets that support life at the centers of every Local Galactic Cluster (LGC) just as it does in our Milky Way Local Galactic Cluster (MW-LGC).

be). Universe is not a "grand design", because there is too much useless real state; no grand designer would have created and allocated resources so poorly.

bf). The life on earth is not a design by a super-entity or grand designer; because, no grand designer even with any intelligence would have designed living species in a way that one species has to consume the other species in order to stay alive.

bg). There is/was no grand-designer; there is/was no big-bang (big-nonsense).

bh). Probability is a human invention; nothing in the nature is random or probabilistic; since, the nature is by and large 
incomprehensible to us, we make the assumption of randomness in order simplify and to gain at least some understanding.

bi). The so-called space-time fabric is functionally the same as the medium density, no difference. In reality, the light follows the curvature of the medium density; the light supposedly follows the curvature of the so called space-time fabric.

bj). The radial movement of galaxies takes place within a Local Galactic Cluster (LGC), and the LGC radius $R<c / H_{\max }$; this ensures the radial speed is always less than the speed of light.

bk). If Hubble relationship is true, reversing back time would not converge all the galaxies to a singular point at the same time. They come to contact with each other one by one; not at the same time.

bl). If the Hubble relationship is true, the age of the universe will be theoretically infinite or unbounded since the radial speed of galaxies are proportional to the distance.

$\mathrm{bm})$. If the age of the universe is the inverse of the Hubble constant, then, the age of the universe will be a constant; forever young.

bn). A burst of light cannot travel forever without being frequency shifted out of the visible region due to energy loss along the path; a burst of light is not an Energizer bunny that keeps going, going, going, ... ; How far a burst of light can travel within the visible frequency spectrum is determined by the path degradation.

bo). Visible universe is the maximum distance a burst of light can travel without being frequency shifted out of the visible frequency due to path degradation; visible universe is an observer dependent moving horizon, or personal visible space.

bp). The cosmic background noise is a result of the distant light bursts that are being frequency shifted out of the visible frequency region due to energy loss along the path or path degradation; not some remnants from a big-bang.

bq). The total amount of matter and energy present in the universe is not measurable or knowable; how can you measure the total matter in the universe when you don't know what the universe is; how vast it is and if it has an origin; there is no dark matter or dark energy.

br). One person's visible universe can be another person's cosmic microwave background, and vice versa.

bs). The electromagnetic propagation energy loss creates an observer independent permanent galactic red shift; this permanent red shift does not represent radial movement of galaxies or space expansion; increasing galactic red shift indicates the radial movement of galaxies.

bt). The permanent red shift due to propagation loss limits our visible universe.

\subsection{Light in a New Light}

The light consists of electromagnetic wave bursts of frequency $n e / h, \forall \mathrm{n}=1,2,3, \ldots$ and constant duration, $F=h / e$, where $e$ is the quantum energy, and $h$ is the Plank constant [6]. The frequency spectrum of light is not continuous. The frequency spectrum is quantized, and the frequency resolution is $e / h$. Each burst of electromagnetic wave propagates at the speed of light according to the Maxwell's Equations as a single unit. If an electromagnetic wave burst of frequency $n e / h$ loses energy $m e$, what remains is a burst of frequency $(n-m) e / h$, where $n \geq m$. An electromagnetic burst of energy $e$, loses or get absorbed as a single unit. These bursts are not random particles or photons. Their path is determined by the density gradient of the medium. The speed of the electromagnetic wave is determined by the electromagnetic properties of the medium, permittivity and the permeability. The gravity has no direct effect on the path of light. In the presence of a medium, the gravity creates a density gradient around the gravitational object which leads to a refraction of light, known as gravitational lensing. In the absence of a medium, the gravity has no effect on light, electromagnetic waves, what so ever.

\subsubsection{Light and Relativity}

Light has no mass. The light does not travel relative to a light source or relative to moving bodies $[4,6]$. The light consists of electromagnetic bursts of constant duration. The duration of a burst is a universal constant. Once a burst is out of source, the source has nothing to do with the burst, and the path a burst takes is completely determined by the density gradient of the medium, or lack of it. Light is not relative. Only the things that have mass are relative.

\subsubsection{Red Shifts and Blue Shifts (observer dependent)}

The perceived red shift or blue shift is a phenomenon that is present when an observer is involved. It doesn't matter how a source is moving, there is no red shift or blue shift in the absence of an observer [5,6]. When a source of light is moving, the frequencies of light bursts the source is giving out do not change. However, the movement of source, or the observer, changes the number of bursts an observer receives, thereby giving the impression that the frequency has changed. When a source is moving away relative to an observer, the rate of peaks or troughs an observer receives is diminished indicating a red shift. Similarly, when a source moves toward an observer, relative to the observer, the rate of peaks or troughs an observer receives increased signaling a blue shift. However, in actual fact, the frequency of the source hasn't changed. The red shift or blue shift is an observer phenomenon, an illusion. If there is no observer present, it does not matter which way or what speed a source of light moves, the frequency is unchanged. Since the frequency shift is an illusion, an observer based phenomenon, different observers observe different frequency shift depending on their relative velocities. It doesn't matter how fast a source is moving, there is no change of frequency or a frequency shift until an observer comes into the scene.

\subsubsection{Permanent Red Shift (Observer Independent)}

When light propagates, it undergoes a path energy loss or a propagation loss that is proportional to the distant the light had travelled. The lost of energy from a light burst result in a decrease of the frequency of the burst, and hence a permanent frequency red shift. The larger the distant a burst of light had 
traveled the higher the propagation loss, and hence the larger the red shift. The frequency shift that is caused by propagation loss is always a red shift. This red shift has nothing to do with the observer; it is completely observer independent. This permanent red shift does not indicate the movement of a source away from us, although one might erroneously interpret it in that manner creating whole stream of cascading misinterpretations such as expanding universe, big-bang, multi-verse and etcetera.

The galactic red shift is mainly due to propagation loss; the further the galaxy away the longer the distance a burst of light has to travel and hence larger the propagation loss creating larger red shift. However, an increasing galactic red shift is the result of galactic orbit dilation due to a mass increase of the galaxy; an indication that the galaxy is moving away.

The increasing galactic red shift does not necessarily mean the radial acceleration of the galaxies. Even, if galaxies are moving away at a constant radial speed, increasing galactic red shift should be present due to propagation loss since the radial distance is increasing; however, this change of red shift as a result of change of radial distance may not be very significant since the change in distance is not very significant. We know the galaxies are not moving away at a constant radial speed since the radial speed is proportional to the radial distance. Therefore, increase in galactic red shift is inherent with the orbit dilation due to change of mass of the galaxies. If we are observing an increasing galactic red shift, that is indeed an indication that the galaxies are moving away due to orbit dilation as a result of the change in mass of the galaxies. The presence of constant galactic red shift does not mean galaxies are moving away; a constant red shift is due to propagation loss, and it is proportional to the radial distance of the galaxy.

\subsubsection{Light Is Not Relative}

If the light is relative, it will result in Sheer Electromagnetic (SEM) waves given by [4],

$$
\begin{gathered}
\frac{\partial}{\partial x \prime}\left[\mathrm{E}_{\mathrm{x}}{ }^{\prime}\right]=\frac{1}{C s} \frac{\partial}{\partial t \prime}\left[\mathrm{E}_{\mathrm{x}}{ }^{ }\right], \\
\frac{\partial}{\partial x^{\prime}}\left[\mathrm{B}_{\mathrm{x}}{ }^{\prime}\right]=\frac{1}{C s} \frac{\partial}{\partial t^{\prime}}\left[\mathrm{B}_{\mathrm{x}}{ }^{\prime}\right]
\end{gathered}
$$

where, $\mathrm{E}_{\mathrm{x}}$ ' and $\mathrm{B}_{\mathrm{x}}$ ' are the electric field and magnetic field relative to the inertial frame in the direction of motion $x, u$ is the speed of the inertial frame, $x^{\prime}$ and $t^{\prime}$ are $x$ coordinate and time relative to the inertial frame,

$$
c_{\mathrm{s}}=(1-\eta) \mathrm{c}^{2} / \mathrm{u}
$$

where, $\eta=\left[1-(\mathrm{u} / \mathrm{c})^{2}\right]^{1 / 2}$.

For $\mathrm{u}<<\mathrm{c}$, we have, $\mathrm{n} \cong 1-\frac{1}{2}(\mathrm{u} / \mathrm{c})^{2}$ and hence, $\mathrm{c}_{\mathrm{s}} \cong \frac{1}{2} \mathrm{u}$

However, as the speed of the inertial frame $u$ approaches the speed of light $\mathrm{c}$, the value of $c_{s}$ approaches $c$,

$\lim _{u \rightarrow c} c_{\mathrm{s}}=c$.

Therefore, we have,

$$
\mathrm{c}_{\mathrm{s}}=\left[\begin{array}{c}
\frac{1}{2} u, u<c \\
c, u \rightarrow c
\end{array}\right]
$$

If the light is relative, the speed of light is no longer a constant. The speed of the Shear Electromagnetic (SEM) waves reaches the speed $c$ only when the inertial frame or the object approaches the speed of Transversal Electromagnetic (TEM) waves, $c$.

The non-existence of Shear Electromagnetic (SEM) waves in the nature is also good evidence that the light is not relative. Path of light is absolute; it does not depend on the state of a source. Path of light is determined by the density gradient of the medium and nothing else. The Maxwell's equations are absolute. The Maxwell's equations are not relative. Light does not propagate relative to moving bodies.

\subsubsection{The Gravity Does Not Bend Light}

The gravity exerts an influence only on the objects that have a mass. The light does not have a mass, and hence the gravity does not have any effects whatsoever on light in a vacuum $[5,4]$.

However, in the presence of a medium, the gravity has an indirect influence on light. The gravity creates a density gradient in the medium surrounding the gravitational object. The density of the medium closer to the gravitational object is higher. The density of the medium falls off as the distance from the gravitational object increases. This creates a gravitational lens in the medium surrounding the gravitational object. This change in the density of the medium in the presence of a gravitational object changes the speed of light resulting diffraction or bending of light. One may interpret this as a case of gravity bending light. However, it is not the gravity that is bending light here. If gravity can bend light, it should be able to do that irrespective of whether a medium is present or not. A gravitational object in a vacuum cannot bend light because light has no mass. The gravity can only influence objects that have mass.

The ability of a gravitational object to bend light in the presence of a medium is called gravitational lensing. Though we have the medium of air surrounding the earth, the earth's gravitation is not significant enough to create a gravitational lens around the earth that is strong enough to create a visible diffraction of light near the earth. However, the gravitation of the sun is strong enough to create a density gradient around the sun that can bend light from distance stars. This has indeed been observed many decades ago by Arthur Ellington and his team during an eclipse of the sun [10]. This observation of the bending of light from distance stars has been erroneously used in the past to claim that the gravity bends light. This observation of diffraction of light near the sun only concludes that there is a gaseous medium around the sun, which creates a gravitational lens.

\subsubsection{Relative Electromagnetic Fields Do Not Exist}

The electromagnetic fields relative to an inertial frame are given by $[2,4]$,

$$
\begin{gathered}
\mathrm{E}_{\mathrm{x}}{ }^{\prime}=\mathrm{E}_{\mathrm{x}}, \mathrm{B}_{\mathrm{x}}{ }^{\prime}=\mathrm{B}_{\mathrm{x}} \\
\mathrm{E}_{\mathrm{y}}{ }^{\prime}=\eta^{-1}\left(\mathrm{E}_{\mathrm{y}}-\frac{u}{c} \mathrm{~B}_{\mathrm{z}}\right), \mathrm{B}_{\mathrm{y}}{ }^{\prime}=\eta^{-1}\left(\mathrm{~B}_{\mathrm{y}}+\frac{u}{c} \mathrm{E}_{\mathrm{z}}\right) \\
\mathrm{E}_{\mathrm{z}}{ }^{\prime}=\eta^{-1}\left(\mathrm{E}_{\mathrm{z}}+\frac{u}{c} \mathrm{~B}_{\mathrm{y}}\right), \mathrm{B}_{\mathrm{z}}{ }^{\prime}=\eta^{-1}\left(\mathrm{~B}_{\mathrm{z}}-\frac{u}{c} \mathrm{E}_{\mathrm{y}}\right)
\end{gathered}
$$


where $\left(\mathrm{E}_{\mathrm{x}}, \mathrm{E}_{\mathrm{y}}, \mathrm{E}_{\mathrm{z}}\right)$ and $\left(\mathrm{B}_{\mathrm{x}}, \mathrm{B}_{\mathrm{y}}, \mathrm{B}_{\mathrm{z}}\right)$ are the $(x, y, z)$ electromagnetic field components relative to a stationary frame, and $\left(\mathrm{E}_{\mathrm{x}}{ }^{\prime}, \mathrm{E}_{\mathrm{y}}{ }^{\prime}, \mathrm{E}_{\mathrm{z}}{ }^{\prime}\right)$ and $\left(\mathrm{B}_{\mathrm{x}}{ }^{\prime}, \mathrm{B}_{\mathrm{y}}{ }^{\prime}, \mathrm{B}_{\mathrm{z}}{ }^{\prime}\right)$ are the $\left(x^{\prime}, y^{\prime}, z^{\prime}\right)$ electromagnetic field components relative to the inertial frame moving in $x$ direction at speed $u$ and $\eta=\left[1-(\mathrm{u} / \mathrm{c})^{2}\right]^{1 / 2}$.

These relative electromagnetic fields only exist if the light is relative. Since the light is not relative, these relative fields do not exist in nature. There is no such thing called "Lorentz force".

\subsubsection{The Range of Light}

A burst of light does not travel forever. There is a maximum distance or a range a burst of light can travel without being frequency shifted out of the visible region, and that range is determined by the path degradation of the medium. The distant light bursts that are being succumbed to path degradation appear as the cosmic microwave background out of the visible frequency spectrum. That is exactly the reason why we cannot see beyond a certain distance into the space, and it is not because we have reached the baby universe or the origin of the universe. The deep space cosmic microwave background diagrams are the representations of the light bursts that have undergone a frequency shift out of the visible frequency region due to path degradation; these bursts are no longer in the visible region of the electromagnetic spectrum, and they still carry the same information about the distant stars that we can't see.

What we see when we look into deep space is NOT our own past, it is our neighbor's.

\subsection{The Time}

The time is a definition. The time is defined based on the changing environment. The time is unique. The time is non-directional. The time is continuous. One can travel neither forward nor backward in time. You can't reverse the time as much as you can't leap forward in time. You can't control time; you can only control what you do with time. It is always the time that travels, not you; you can let the time pass if you want, or you can waste it by working on multi-verse, or inflation. The idea that you can stay young by traveling faster is simply ridiculous and utter nonsense.

\subsubsection{Time Is Not Relative}

The relative time for a given inertial frame of constant speed $u$ in the direction $x$ is given by $[4,5]$,

$$
\begin{gathered}
\mathrm{t}_{\mathrm{x}}{ }^{\prime}=\mathrm{n}^{-\mathrm{n}} \mathrm{t} \\
\mathrm{t}_{\mathrm{y}}{ }^{\prime}=\mathrm{t} \\
\mathrm{t}_{\mathrm{z}}{ }^{\prime}=\mathrm{t}
\end{gathered}
$$

where, $\mathrm{\eta}=\left[1-(\mathrm{u} / \mathrm{c})^{2}\right]^{1 / 2}, n$ is an integer $0<\mathrm{n}<\infty, \mathrm{t}_{\mathrm{x}}{ }^{\prime}, \mathrm{t}_{\mathrm{y}}{ }^{\prime}, \mathrm{t}_{\mathrm{z}}{ }^{\prime}$ are the $x, y, z$ component of relative time $t^{\prime}$, and $t$ is time.

If the time is relative, the relative time depends on the speed of the inertial frame, and hence the relative time will be directional. A directional velocity produces a directional relative time. The relative time on the direction of motion of the inertial frame will be different from the relative time on the lateral plane perpendicular to the direction of motion. In addition, the relative time is not unique since relative time is a function of $n$, and $n$ is an integer that can have an infinite number of values, $0<n<\infty$.

The time has to be non-directional. The time can't be directional. The time must be unique. Therefore, time cannot be relative. The time is absolute. The time is same everywhere in the universe irrespective of the observer's state of being. The motion has no effect on time.

\subsubsection{There Is No Space-Time}

If the time is relative, the space-time function of an inertial frame at speed $u$ in the direction of motion $x$, is given by [4], $t_{x}{ }^{\prime}=\eta^{-n}\left(t-u x / c^{2}\right)$, where $\eta=\left[1-(u / c)^{2}\right]^{1 / 2}, n$ is an integer $0<n<\infty$. The space-time $t^{\prime}$ is directional. Further, the space time function is not unique since $n$ can take any integer value. The time, however, can't be directional. The time must also be unique. Hence the space-time cannot exist. There is no such thing called space-time.

\subsection{The Relativity}

The motion of any object that has a mass is relative and depends on the observer's frame of reference. However, "non-things" that do not have mass are not relative. "Things" that have mass are relative. The electromagnetic energy does not have a mass and hence the propagation of electromagnetic waves is not relative. Since the speed of light is a constant that is determined by the electrical properties of the medium, a moving object contracts in all directions.

\subsubsection{Special Relativity Does Not Hold True}

The Special Relativity $[1,10]$ is based on the premise that the propagation of light is relative. It also considers the time to be relative. We know that the light is not relative and the time is not relative $[4,5]$. The propagation of light is absolute. The time is absolute. Hence, the special relativity does not hold true.

\subsubsection{General Relativity Does Not Hold True}

The General Relativity [10,2] uses the Special Relativity as its foundation. When the Special Relativity is not true, it is obvious that the General Relativity does not hold true either. In addition, there are several more reasons why the General Relativity does not hold.

i) The General Relativity is based on the idea that the concept of space-time exists. As we have seen, if space-time exists, then, the space-time is not unique because the space-time function is not unique [4]. Further, space-time is directional; the space-time along the direction of motion is different from the relative time on the lateral plane perpendicular to the direction of motion. The time can't be directional. Time must be non-directional. Time must be unique. So, there is no space-time [4]. Space-time does not exist. In a moving body, the time does not depend on the space. Time does not depend on the motion of an object. A quantity that depends on the velocity will be directional since the velocity is directional.

ii) The General Relativity is also based on the idea of the 
Principle of Equivalence. The Principle of Equivalence states that "an object at rest on a surface of a gravitational object with gravity $g$ is the same as an object moving at an acceleration $\alpha=g$ ". If this is true, this has to hold all the time. However, as time $t$ approaches $\mathrm{t}=(\mathrm{c}-\mathrm{u}) / \alpha$, the accelerating object reaches speed of light and will turn itself into a transient black-hole. The object at rest on a gravitational surface of gravity $g$ remains the same, and remains at rest irrespective of time for eternity. Further, the physical laws do not apply for the accelerating object when time reaches $\mathrm{t}=(\mathrm{c}-\mathrm{u}) / \alpha$. Therefore, the General Relativity does not hold true.

\subsection{The Space}

Although time is a definition, space is not a definition. Space is a three dimensional "non-thing" that is continuous. Unlike time, space exists and therefore one can travel in space in any direction one wants. In the case of time, it is always the time that travels, never you. However, in space, it is always you that travel, never the space. Space does not depend on time. The time does not depend on space. Space does not expand or contract. The gravity has no effect on space since space has no mass. If we do not like the space we are in, we can move. However, we can't do this with time; if we do not like the time we are in, we just have to let the time pass; we can't move to a different time.

\subsubsection{Space-Time Fabric Is Just a Different Name for the Medium Density}

From the outset, it appears that what is referred to as the space-time fabric is just another name for the density of the medium. In the absence of a medium or in a vacuum, the density is zero. We can observe the similarities between the two in all aspects:

i) In the absence of a medium, light follows a straight path. However, in the presence of a medium, the light follows the density gradient of the medium, or the curvature of the medium. The light is also considered to follow a straight path when the curvature of the space-time fabric is zero; the light follows the curvature of the so called space-time fabric, or the gradient of the space-time fabric.

ii) The density gradient increases as it gets closer and closer to a gravitational object. Similarly, in the case of the so called space-time fabric, the negative gradient of the space-time fabric increases as it gets closer to a gravitational object.

iii) In the presence of a medium, a gravitational object creates ripple in the density of the medium. Similarly, a gravitational object is considered to create ripple in the space-time fabric.

iv) In the presence of a medium, the light bends near a gravitational object. The light is also assumed to bend near a gravitational object in the presence of so called space-time fabric.

v) When an object is free to move in space, it is said to follow the negative curvature of the space time fabric. It is also true that an object that is free to move also follows the positive curvature of the medium density or gravity. Only difference seems to be the sign of the curvature.

Since the light is not relative, there is no space-time [4]. Although there is no space-time as per se, what is referred to as the space-time fabric is simply appears to be the negative medium density; on the outset, functionally, so called space-time fabric is just another name for the negative medium density.

\subsubsection{The Universe Is Not Expanding}

The light from nearby galaxies is observed to have an associated increasing red shift $[8,10]$. This increasing red shift can be a combination of the observer dependent red shift due to radial movement of the galaxies as well as the permanent or observer independent red shift due to propagation loss. Since some of the galaxies are showing a blue shift indicating that they are moving toward us, and we are dealing with nearby galaxies, the observed increasing red shift cannot be considered as a result of the path energy loss alone. The increasing red shift indicates that the galaxies are moving away from us. This observation, together with a theoretical justification from the General Relativity $[2,10]$, have used in the past to make an erroneous conclusion that the universe is expanding, an idea that the space itself is expanding. The idea of expanding universe is simply preposterous. This false notion of expanding universe also led to the concept of big-bang [10], the idea that the universe started from a singular event at a singular point called a big bang, the birth of universe. The concept of expanding universe in reverse has also been used to calculate the age of the universe as 13.5 billion years [10,9].

We now know that the light is not relative, the propagation of light is absolute [4]. And also, it is clear that the principle of equivalence that the General Relativity is based on does not hold true. Therefore, neither the Special Relativity nor the General Relativity hold true. This means that there is no theoretical justification for the idea of expanding universe. The universe is neither expanding nor contracting [7]. There was/is no big-bang. The idea that the universe is 13.5 billion years old was calculated under the assumption that the universe is expanding. This age of the universe has no meaning when there is no expansion of universe. What is equally preposterous is to suggest that the deep space temperature variations, and the thermal noise that appear on an off-tuned television as the remnant from the big-bang; are we out of our minds? It is not necessary to have a big-bang to have deep space temperature variations. At any given moment, the amounts of electromagnetic wave bursts that pass through different points in deep space are different resulting temperature variations in the deep space. The deep space temperature variations are always there due to the crisscrossing of electromagnetic bursts in different amounts; it is not a result of a big-bag. The light we receive from distance is our neighbors'. What we see at distance is not our own past, but our neighbors'.

The galactic red shift we are observing $[8,10]$ is a result of 
galactic orbit dilation [7]. The mass of any object in the universe is not a constant; mass is time varying. When a mass of a galaxy is increased, its orbit dilates giving the galaxy a radial velocity, making it appears as if the galaxy is moving away. If a galaxy loses its mass, the orbit of the galaxy contracts resulting in a galactic blue shift, making it appears as if the galaxy is moving toward us. In the case of planets, what prevails is a mass loss and hence the orbit contraction, resulting a movement toward the sun leading to global warming. A collision of a planet with a meteorite or asteroid leads to an abrupt dilation of planetary obit. The abrupt orbit dilation leads to an abrupt planetary cooling.

\subsubsection{Are We at the Center of the Universe}

Many centuries ago it was considered to be so. Today, the question itself is considered to be nonsense. Of course, we are not at the center of the universe. However, when we direct our telescopes in the direction of other nearby galaxies, we find that some of the galaxies are moving away from us while some other galaxies moving toward us. This indicates that the some of the nearby galactic orbits are dilating and some of the galactic orbits are contracting. So, it must be that our nearby galaxies are orbiting around our Milky Way galaxy. In other words, our Milky Way galaxy is at the center of a galactic cluster where the other galaxies in the cluster are orbiting around.

Every galaxy, in fact, every object in the universe, must have its own speed and orbit in order to remain free in the space; speed is the currency in the universe. Any object that does not have speed will get absorbed by a larger object due to gravity that is ever present everywhere in the universe; there is no place that is free of gravity in space. Our galactic cluster centered on the Milky Way must orbit around even a bigger galactic cluster.

Our observation that the nearby galaxies are either moving away from us or moving toward us indicates that the masses of the galaxies in our Milky Way Local Galactic Cluster are either increasing or decreasing. The increase in mass leads to orbit dilation indicating that these galaxies are moving away from us while the decreasing mass leads to orbit contraction indicating that these galaxies are moving toward us, which means we are at the orbiting center of this galactic cluster. If the nearby galaxies in our local galactic cluster are moving away from the Milky Way galaxy, this may also occur due to the increase in the mass of the Milky Way galaxy where the other galaxies are orbiting around. If the distance to the $i^{\text {th }}$ galaxy from the Milky Way galaxy is $r_{i}$, and the mass of the $i^{\text {th }}$ galaxy is $m_{i}$, then the orbit dilation relationship is given by,

$$
u_{i}(t)=H_{i}(t) r_{i}(t)
$$

where, $\mathrm{H}_{\mathrm{i}}(\mathrm{t})=2 \frac{\partial}{\partial t} \ln \left[\mathrm{m}_{\mathrm{i}}(\mathrm{t})\right], \mathrm{u}_{\mathrm{i}}(\mathrm{t})=\frac{\partial}{\partial t}\left[\mathrm{r}_{\mathrm{i}}(\mathrm{t})\right]$, and $\mathrm{t}$ is the time.

Not only galactic orbits are moving away from us, they are moving away at an accelerated rate.

The orbit acceleration $\frac{\partial}{\partial t}\left[\mathrm{u}_{\mathrm{i}}(\mathrm{t})\right]$ is given by,

$$
\frac{\partial}{\partial t}\left[\mathrm{u}_{\mathrm{i}}(\mathrm{t})\right]=\psi_{\mathrm{i}} \mathrm{r}_{\mathrm{i}}(\mathrm{t}), \text { where, }
$$

$$
\begin{gathered}
\psi_{\mathrm{i}}(\mathrm{t})=\mathrm{H}_{\mathrm{i}}^{2}(\mathrm{t})+\frac{\partial}{\partial \mathrm{t}} \mathrm{H}_{\mathrm{i}}(\mathrm{t}), \\
\psi_{\mathrm{i}} \cong\left[2 / \mathrm{m}_{\mathrm{i}}(\mathrm{t})\right] \frac{\partial}{\partial t} \frac{\partial}{\partial t}\left[\mathrm{~m}_{\mathrm{i}}(\mathrm{t})\right], \forall \mathrm{i}=1,2, \ldots .
\end{gathered}
$$

The orbit dilation relationship is the same as the Hubble relationship $[8,10]$ except that in Hubble relationship $\mathrm{H}$ is assumed to be a constant and known as Hubble Constant. In actual fact, $\mathrm{H}$ is a time varying quantity that depends on the change of mass of a galaxy.

So, there is no doubt that we are at the center of a galactic cluster. The rest of the galaxies in our galactic cluster are orbiting around the Milky Way galaxy. The changes of mass of the galaxies give these galaxies a radial movement from us at an accelerated rate. The radial speed the nearby galaxies moving away from us depend on the rate of change of mass of the galaxies. The increase of the mass of the Milky Way galaxy can also make the nearby galaxies in our cluster to move away from us. If the mass of a galaxy in our Local Galactic Cluster (LGC) is decreasing, the orbit of that galaxy will be contracting proportional to the rate of change of mass, and to the radial distance of the galaxy from the orbiting center, the Milky Way Galaxy in our Local Galactic Cluster. Since the orbit dilation is proportional to the radial distance of the galaxy from the orbiting center, the orbit dilation always takes place at an accelerated rate [7].

Although we are not at the center of the universe, we are indeed at the center of a local galactic cluster where nearby galaxies are orbiting around us, the Milky Way Galaxy. However, there is no reason to assume that a blue planet that supports life can exist only at the center of our Local Galactic Cluster (LGC) that is centered at Milky Way Galaxy. It is possible that there are blue planets that support life at the centers of all the rest of the Local Galactic Clusters (LGC). In fact, there must be observers at the center of another galactic cluster observing the same galactic red shift due to radial galactic movement and thinking that they are indeed at the center of their galactic cluster; in fact, at one time, those observers might have thought they were at the center of the universe as we did many centuries ago. However, we are not at a realistic communicating distance with those other observers at the centers of the other galactic clusters. In addition, the languages of those other galactic intelligent life could be quite different from ours that it may appear to us as just noise making distant communication further impossible. Could it be that "the life can only exists on a planet at a center of a galactic cluster, and there could be blue planets that support life at the centers of all the other galactic clusters"?

\subsubsection{The Mass of the Earth Is Not a Constant}

If you think that the mass of the earth is a constant, you may want to reconsider that thought. Start asking this question. What is the most abandon material in the universe? The most abandon material in the universe is hydrogen. Most compound, organic as well as non-organic compound, on earth contains hydrogen. Yet, there is almost no hydrogen in the air in its natural form as a gas. Generate hydrogen whatever means available and release it into the air. It doesn't matter how much hydrogen you release into the air, there will not be any increase in the hydrogen content in the air. Why is that? The 
earth's gravity is not strong enough to retain hydrogen as an independent gas. The hydrogen gas will leak out of the atmosphere resulting in a mass loss.

Contrary to the current belief, the mass and electromagnetic energy are not one and the same. Electromagnetic energy has no mass. However, the mass and electromagnetic energy are convertible to each other. We convert mass to electromagnetic energy by burning fossil fuels. The nature converts mass into electromagnetic energy by atomic decay or radiation. Once the mass is converted into electromagnetic energy, the end result is a mass loss. On the other hand, the biomass converts the electromagnetic or light energy into mass. So, the mass loss and the mass gain are in a natural equilibrium. However, the excessive human intervention through the increase fossil fuel extraction and consumption, and the destruction of the biomass could tip the natural balance toward a mass loss.

So the mass of earth is not a constant. Mass of the earth is time varying. Due to excessive human intervention, the mass of the earth is decreasing steeply. The rate of change of mass of the planet plays a crucial role in the sustainability of the life on earth; it determines temperature variations of a planet.

\subsubsection{Total Mass and Energy in the Universe}

The universe is not a result of a big-bang. The origin of the universe is unknown. There is no reason to believe the size of the universe is finite. It is not possible to measure the amount of matter and energy of the universe. It preposterous to claim we know how much matter and energy there is in the universe when we even don't know how vast the universe is. There is no dark matter of dark energy in the universe; what is there is our inability to measure the amount of matter and energy in the universe, and our ignorance and our reluctance to accept that fact.

\subsubsection{Visible Universe}

There is a maximum limit or a range to the distance any burst of light can travel in the visible region of the spectrum, and it is determined by the path degradation or path energy loss. A burst of light cannot travel forever in the visible region of the spectrum due to path energy loss; it is a fact of nature.

Definition: The Range of Light

The range of light is the maximum distance a burst of light can travel in the visible region without being shifted out of the visible region of the electromagnetic spectrum due to path degradation.

The range of light is not infinite. The limited range that light can travel in the visible region of the spectrum is the reason why we cannot see deep into the space beyond a certain distance, not because the baby universe or the origin of the universe is reached. The visible universe is the maximum distance a burst of light can travel without being succumb to the path degradation, and it has nothing to do with the origin of the universe. The visible universe is an observer dependent moving horizon. If the range of a light burst is $r$ light years, then I have a visible universe of radius $r$ centered on me, and somebody else in another planet may have a visible universe of radius $r$ centered on him or her. When a person moves, that person's visible horizon is moving with that person. This is a good indication that the universe is not finite. The visible universe has no bearing what so ever on a hypothetical big-bang. The light bursts that are out of the visible frequency range of the electromagnetic spectrum due to path degradation manifest themselves in the microwave region as the cosmic microwave background. The deep space cosmic background diagrams represent the light bursts that have already undergone path degradation; they carry the same information just like visible light. If light had had an infinite range, i.e. the ability to travel infinite distance without being shifted out of the visible frequency range of the electromagnetic spectrum, we would not have come across a cosmic microwave background. The visible range of light is finite and therefore we get to experience the cosmic background noise. The cosmic background noise is not some left over or remnant from a hypothetical big-bang. The microwave cosmic background noise Penzias and Wilson measured in 1964 represents the light bursts that have undergone this frequency shift out of the visible range of the spectrum due to path degradation; it is not a remnant from a big-bang as they seem to proclaim.

\subsubsection{The Deep Space Temperature Maps (Cosmic Microwave Background)}

The deep space temperature maps do not represent the origin of the universe, a baby universe or a big-bang. The deep space temperature maps are representations of the light bursts that are being frequency shifted out of the visible frequency region due to path degradation or energy loss.

The light consists of electromagnetic wave bursts of frequency $n e / h, \forall \mathrm{n}=1,2,3, \ldots$ and constant duration, $F=h / e$, where $e$ is the quantum energy, and $h$ is the Plank constant [6]. Each burst of electromagnetic wave propagates at the speed of light according to the Maxwell's Equations as a single unit. If an electromagnetic wave burst of frequency ne/h loses energy $m e$ along the path when it travels a distance of $r$ light years, what remains is a burst of frequency $(n-m) e / h$, where $n \geq m$. An electromagnetic burst of energy $e$, loses or get absorbed as a single unit. Consider that the lowest frequency of the visible band is $f_{L}$, and the highest frequency of the visible band is $f_{H}$, then, $f_{L}=n_{L} e / h$, and $f_{H}=n_{H} e / h$ where $n_{L}$ and $n_{H}$ are integers. Now, if $\left(n_{H^{-}}-m\right) e / h=\left(n_{L}-1\right) e / h$, then the distance $r$ will be the range of the visible universe. Further, if $\left(n_{H^{-}} m\right) e / h<n_{L} e / h$, then a light burst of frequency $(n-m) e / h$ will be out of the visible region contributing to the cosmic microwave background. So, the light burst of frequency $n e / h$ will be out of the visible region of the frequency spectrum due to energy loss incurred during the propagation of distance $r$, and it is no longer a visible light burst; instead, it appears in the form of the cosmic microwave background. One person's visible universe can be another person's cosmic microwave background. When we are measuring the deep space temperature variations, what we are measuring is the presence of this frequency shifted light bursts that are no longer in the visible region of the spectrum due to energy loss along the path, not some remnant of a hypothetical big-bang. The information we get from cosmic microwave background is available within the visible region for someone elsewhere at a distant due to the observer dependent horizon of the visible universe.

\subsection{Back to Earth}


Certain global events are shaped by the location of the orbit of the earth. The location of the orbit of the earth, in effect, is determined by the rate of change of mass of the earth. Our actions influence the rate of change of mass of the earth to a certain degree.

\subsubsection{What Causes the Global Warming}

There is no denying that the green house gases cause global warming. However, there is another more prominent side to the cause of global warming. We know that the human intervention through excessive fossil fuel consumption and the destruction of the biomass has tipped the natural mass stability of the earth toward a net mass loss. When the mass of the earth is decreasing, the rate of change of the mass of the earth will lead to the orbit contraction. The rate of change of mass of the earth gives the earth a radial velocity and acceleration toward the sun, in effect, bringing the earth closer to the sun resulting in Global Warming. The rate of change of mass determines rate of Global Warming. Ultimately, how fast we tip the natural balance between the mass increase and mass loss toward a net mass loss determines the severity of the Global Warming.

\subsubsection{Hydrogen Fuel Is Not Environmentally Friendly}

We always thought Hydrogen fuel cells as an environmentally friendly alternative to our energy needs. We have seen a surge of Hydrogen fuel cell driven automobiles coming into the market. This thinking that the Hydrogen fuel is an environmentally friendly alternative to our energy needs may require serious re-evaluation.

If you think by switching to Hydrogen powered automobiles, and Hydrogen powered energy sources, you will save the planet from Global Warming, think again. Any hydrogen leaked into air is going to be permanently loss from earth because the gravitation of the earth is not strong enough to keep hydrogen as a gas tied to earth. The leaked out Hydrogen result in a mass loss of the earth. The rate of mass loss creates orbit contraction contributing to Global Warming. Aside from the noble intentions, the use of Hydrogen fuel cells may accelerate the Global Warming. Any hydrogen that is released into the air can be considered as a permanent loss of it; it is not wise to lose something that there is no way to gain, doesn't matter how hard we try. The only way for earth to gain any new hydrogen is through a collision with meteorite or asteroid.

\subsubsection{The Cause for the Extinction of Dinosaurs}

It has been said that the extinction of the biggest animal on earth, dinosaurs, was a result of a giant asteroid collision with the earth. A large crater that is believed to be the result of that collision has also been located near Yucatan Peninsula in Mexico. It is also said that the impact of the collision and the dust cloud that resulted by it wiped out the Dinosaurs as well as most of the other species.

However, it may not be the dust cloud or the impact that brought the doom to the plant and animal, especially to the Dinosaurs. If the asteroid had been as massive as it was claimed to be, then the collision might have increase the mass of the earth at a very short interval of time; it might have changed the axial tilt too. This increase of mass would have given the earth an outward radial velocity causing the orbit of the earth to expand. It is not the size of the asteroid that determines the strength of the radial velocity or orbit dilation; it is the rate of change of mass of the earth that determines the value of the radial velocity. The orbit dilation or the radial speed to rate of mass increase is given by,

$$
u(t)=H(t) r(t)
$$

where, $\mathrm{H}(\mathrm{t})=2 \frac{\partial}{\partial \mathrm{t}} \ln [\mathrm{m}(\mathrm{t})], \mathrm{u}(\mathrm{t})=\frac{\partial}{\partial \mathrm{t}}[\mathrm{r}(\mathrm{t})]$, and $m(t)$ is the mass of the earth, $t$ is the time. The orbit dilation or contraction takes place at an accelerated rate.

The orbit acceleration $\frac{\partial}{\partial t}[\mathrm{u}(\mathrm{t})]$ is given by,

$$
\begin{gathered}
\frac{\partial}{\partial t}[\mathrm{u}(\mathrm{t})]=\psi \mathrm{r}(\mathrm{t}), \\
\psi(\mathrm{t})=\mathrm{H}^{2}(\mathrm{t})+\frac{\partial}{\partial t} \mathrm{H}(\mathrm{t}), \psi(\mathrm{t}) \cong(2 / \mathrm{m}(\mathrm{t})) \frac{\partial}{\partial t} \frac{\partial}{\partial t}[\mathrm{~m}(\mathrm{t})],
\end{gathered}
$$

where, $r(t)$ is the distance to the earth from the sun, and $m(t)$ is the mass of the earth at time $t$.

The collision of earth with an asteroid is so sudden that it must have increased rate of change of mass $\partial[m(t)] / \partial t$ tremendously creating abrupt orbit dilation and bringing with it a sudden ice age or extremely low temperatures, the largest animals such as dinosaurs and many other species could not cope. The cooling might have been so extreme and sudden that most species, especially the mammoth animals, could not have been able to adopt. The change is so drastic that the most species did not have sufficient time to adopt genetically. Only the small creatures, creatures that could survive inside underground burrows, might have been able to survive such changes.

It is not necessary for the asteroid to be massive for the earth to undergo orbit dilation. The orbit dilation is proportional to the rate of change of mass. Even a moderate size asteroid collision with earth can create a significant outward radial speed since the collision takes place in a very small time interval. The rate of change of mass, $\partial[m(t)] / \partial t$, in an impact would be massive. After the impact of earth with the asteroid, the natural gradual reduction of mass of the earth might have shifted the orbit inward with time bringing with it moderate temperatures for sustainable life.

That was then; an extreme cooling of the earth due to an extrinsic activity, a collision of the earth with a massive asteroid, that eliminated the massive living creatures and many other species completely. Are we experiencing the complete opposite of it today; an extreme warming due to steep mass loss? If the orbit contraction is so significant, the species may not have sufficient time for genetic adaptation to high temperatures to ensure the survival of the species. Unlike the destiny of the dinosaurs that was created externally by nature, today, in our case, we may be creating our own extinction intrinsically. However, a collision of earth with an asteroid is something that we can hope for since such a collision brings an abrupt cooling due to orbit dilation.

\subsection{Has the Nature Got It Wright}


If the Global Warming reaches a level that the environment for life on earth is not favorable, then, an impact with an asteroid would not be a bad thing. So, once in a while arrival of an asteroid toward the earth, putting the earth in a collision course with an asteroid, might be the nature's way of making sure that the earth remains as a place that supports life in long term, even though such a collision may create short term havocs. Nature seems to know what it is doing especially because there is no higher entity with authority to tell the nature what it is doing wrong. If the nature has a way to figure out what it is doing wrong, it should have not created all these useless real state. Just look around, Mercury, a useless rock, Venus useless rock, Mars, Jupiter, Saturn, Uranus, Neptune, Pluto, and many other planets and galaxies, they are either useless rocks or deadly gas balls, where no life can exist. And also, why would the nature create species in a way one species had to gobble up the other to remain alive. Just look around, whether on land, in the air, or in the water, everywhere, work place too, every living thing spend their life keeping the mouth open looking for ways to gobble up the other no matter what. Why should the survival of a lion depend on the death of a dear? This applies to humans too. If some creator-entity, a grand designer, has created the universe, why should any entity or a grand designer creates the universe in such a nonsensical manner? We wouldn't have created so much junk real state, and the life in a way one species had to consume other in order to sustain life, if we were granted the task of creating a universe. It shows that there had never been a grand designer or creator-entity, because no grand designer, even with a little bit intelligence, would have created so much useless real state. No grand designer or creator entity, even with a little bit of intelligence, would have created species in a way that one has to eat the other for survival; not to mention how cruel that grand designer or creator-entity should be to create species in such a manner. It shows that the universe did not come to existence through an out of sight creator-entity or a grand designer. To suggest that the universe is a result of expanding and contracting space and a big-bang is equally bizarre and unrealistic as much as the creation by an invisible grand designer or creation-entity. How did the universe come in to being? One thing is clear; it is not through a bizarre and nonsensical hypothetical big-bag or any other equally bizarre and nonsensical, non-existent, invisible grand designer or creator-entity.

\section{Age of the Universe}

Everywhere we look, we hear that the universe is approximately 13.5 billion years old. How did they derive this figure? This figure had been derived using the Hubble relationship,

$$
\mathrm{u}=\mathrm{Hd}
$$

where, $u$ is the receding speed of a galaxy, $d$ is the distance to the galaxy and $H$ is the Hubble constant.

If we take the $n^{\text {th }}$ differential of the Hubble relationship given in eqn. (13), we have, $\partial^{\mathrm{n}}(\mathrm{u}) / \partial \mathrm{t}^{\mathrm{n}}=\mathrm{H}^{\mathrm{n}} \mathrm{d}$.

When $n=2$, we have the radial acceleration

$$
\partial^{2}(\mathrm{u}) / \partial \mathrm{t}^{2}=\mathrm{H}^{2} \mathrm{~d}
$$

So, the galaxies move away from us always at acceleration.

Using this relationship, the time $T$ taken for the galaxy to travel distance $d$ is obtained as $[9,10]$,

$$
\mathrm{X}=\mathrm{d} / \mathrm{u} \text {. }
$$

Substituting for $u$ from equation (13),

$$
\mathrm{T}=1 / \mathrm{H} \cong 13.5 \text { billion years. }
$$

Even though the distances to the galaxies are different, the $T$ is the same for all the galaxies since $d_{i} / u_{i}=1 / H, \forall i=1,2,3, \ldots$, where, $i$ is the $i^{\text {th }}$ galaxy. In other words, all the galaxies started receding from a singular point some 13.5 billion years ago. Using this calculation based on the observed Hubble constant, estimated age of the universe is said to be 13.5 billion years. The universe is said to be started from a singular point some 13.5 billion years ago. However, since $H$ is a constant, $1 / H$ is a constant. From eqn. (15), the age of the universe $T=1 / H$, and therefore, the age of the universe is a constant. The universe has an age that never varies with time. It doesn't matter when you calculate the age of universe, the age of the universe always remains at 13.5 billion years. If we had calculated the age of the universe 10 billion years ago, the age of the universe is 13.5 billion years. Similarly, if we calculate the age of the universe 10 billion years from today, we still get the age of universe as 13.5 billion years. We have a universe that never ages; it always remains 13.5 billion years young; forever young! Isn't that interesting?

There is a major error in this calculation. The receding speed is not a constant. A galaxy did not move at a constant speed in that $d$ distance it traveled. The galaxies do not have constant speeds. So, the time $T$ taken for a galaxy to travel distance $d$ cannot be obtained simply by dividing distance $d$ by the instantaneous speed $u$ at distant $d$.

Theorem 10.1: Impossibility of Big Bang

If the Hubble relationship hold true, big-bang is not possible; in other words, it is not possible for the universe to start from a singularity.

Proof: Consider a galaxy at distance $d$ that is receding at speed $u_{d}$. When the galaxy is at distance $r$ where $r \leq d$, it has the receding speed $u_{r}$. According to the Hubble's law,

$$
\begin{aligned}
\mathrm{u}_{\mathrm{d}} & =\mathrm{Hd} \\
\mathrm{u}_{\mathrm{r}} & =\mathrm{Hr}
\end{aligned}
$$

When the galaxy is at distance $r$, during a small time interval $\Delta t$, the distance galaxy recedes $\Delta r$ is given by,

$$
\Delta \mathrm{t}=\Delta \mathrm{r} / \mathrm{u}_{\mathrm{r}}
$$

When $\Delta r$ approaches zero, we get, $\lim _{\Delta r \rightarrow 0} \Delta t / \Delta r=\partial t / \partial r$ and hence,

$$
\partial \mathrm{t} / \partial \mathrm{r}=1 / \mathrm{u}_{\mathrm{r}}
$$

Substituting for $u_{r}$ in eqn. (19) from eqn. (17), we get,

$$
\partial \mathrm{t} / \partial \mathrm{r}=(1 / \mathrm{H}) \frac{1}{r}
$$




$$
\begin{aligned}
& \partial \mathrm{t}=(1 / \mathrm{H}) \frac{\partial r}{r} \\
& \mathrm{t}=(1 / \mathrm{H}) \ln [\mathrm{r}]+\varphi
\end{aligned}
$$

where $\varphi$ is a constant.

If the galaxy takes time $T_{r}$ to reach distance $r$ and time $T_{d}$ to reach distance $d$, we have,

$$
\mathrm{T}_{\mathrm{d}}-\mathrm{T}_{\mathrm{r}}=(1 / \mathrm{H}) \ln [\mathrm{d} / \mathrm{r}]
$$

This relationship holds for any galaxy when it travels from distance $r$ to distance $d$. For the $i^{\text {th }}$ galaxy, we have,

$$
\mathrm{T}_{\mathrm{di}^{-}}-\mathrm{T}_{\mathrm{r}}=(1 / \mathrm{H}) \ln \left[\mathrm{d}_{\mathrm{i}} / \mathrm{r}\right]
$$

$T_{d i}-T_{r} \neq T_{d j}-T_{r}$, since $d_{i} \neq d_{j}, \forall i \neq j$.

They do not merge to a singular point if the time reverses; they meet each other one by one in a queue. Therefore, if the Hubble relationship holds true, the galaxies cannot collapse to a single point together; there is no singularity and hence a big bang is not possible if the Hubble's relationship holds true.

Lemma 10.1: Infinite Age of the Universe

If the Hubble relationship holds, the age of the universe is infinite.

Proof: From eqn (23), we have,

$$
\mathrm{T}_{\mathrm{d}}-\mathrm{T}_{\mathrm{r}}=(1 / \mathrm{H}) \ln [\mathrm{d} / \mathrm{r}]
$$

When $r$ approaches zero, $\mathrm{T}_{\mathrm{r}}=0$ and, we have the age of the universe $X=T_{d}$, and hence,

$$
T=(1 / H) \lim _{r \rightarrow 0} \ln [d / r] \text {, i. e. } T=\infty .
$$

If the Hubble relationship holds, the age of the universe will be theoretically infinite or unbounded.

Since there was no big-bang, there is no remnant from a big-bang either; you can't call cosmic microwave background (frequency shifted light bursts due to path energy loss) a remnant of a hypothetical big-bang. What you see on the screen of an off-tuned television set is thermal noise that any electronic circuit generates, and calling it a remnant of a big-bang is simply preposterous. The light we receive from the distance is not our past but our neighbors', distant galaxies, galactic clusters, super clusters, etcetera.

\section{Conclusions}

The Maxwell's equations are not relative. The propagation of light is not relative. It doesn't matter what state a source is, it could be at stand still, moving at a constant speed, or accelerating, the light does not propagate relative to a source. A source of light releases light in wave bursts; the duration of a burst is a universal constant. Once a light burst is out of a source, its propagation has nothing to do with the source. The path a burst of light takes is completely determined by the density gradient of the medium.

Time is not relative. Time is absolute. Mass of an object is not relative. Mass is absolute. There is no relativistic mass. The Special Relativity does not hold true. If the time depends on the observer's speed, then the relative time will be directional since the speed is directional. The relative time along the direction of the motion of the object will be different from the relative time in the lateral plane perpendicular to the direction of motion of the object. Relative time of an object could be non-directional if and only if the speed of the object is Omni-directional; however, there is no such thing called Omni-directional speed. It is noteworthy that and object with an Omni-directional speed is an object at stand still. Further, the relative time is not unique. So, time can never be relative. Only the things that have mass are relative. Light does not have a mass; light is not relative. Time does not have a mass; time is not relative.

If the light is relative, the speed of light is no longer a constant. If the light is relative, it will result is Shear Electromagnetic (SEM) waves that would travel at a much slower speed that depends on the speed of the inertial frame. Only when the speed of the inertial frame or the object reaches the speed of the Transversal Electromagnetic (TEM) waves, $c$, the speed of the SEM waves reaches $c$. In fact, there is no such thing called Shear Electromagnetic (SEM) waves in the nature. The non-existence of SEM waves in the nature is a good testimony that the light is not relative.

If the time is relative, the relative time will become dependent of the space giving rise to space-time. The space-time is directional and not unique. Since time must be non-directional and unique, there is no such thing called space-time. Without a space time, there will not be a Theory of General Relativity either. Further, the General Relativity is based on the principle of equivalence, which states that "a body standing on a gravitational object of gravity $g$ is equivalent to an object moving at acceleration $\alpha=g$ ”. If the equivalence principle holds true, it has to hold true for all the time. However, a moving body at acceleration $\alpha=g$ reaches the speed of light when time reaches $t=(c-u) / \alpha$, where $c$ is the speed of light, and $u$ is the inertial speed. When time reaches $t=(c-u) / \alpha$, the volume of the accelerating object reaches zero and the mass density will become infinity or unbounded. In other words as time reaches $t=(c-u) / \alpha$, the accelerating body turn itself into a transient black hole. The physical laws no longer hold true for an accelerating body when time $t$ reaches $t=(c-u) / \alpha$. At the same time, the object resting on the ground of gravity $g$ remains intact, and it is in compliance with all the physical laws all the time. Therefore, the General Relativity does not hold true. Since the General Relativity is the only theoretical justification to the claim that the space is either expanding or contracting, in the absence of that theoretical justification, we know that the space is "neither expanding nor contracting".

From the outset, what is referred to as space-time fabric is nothing but the density of the medium. Functionally, they are the same. Light follows the curvature of the medium density. Light is also assumed to follow the curvature of the so called space-time fabric. A gravitational object creates ripples in the medium density. A gravitational object is also assumed to create ripple in the so called space-time fabric. An object in space follows the curvature of the medium density in the space, or in other words gravity; an object moves from low density to a high density or follow the positive curvature of the medium density. In the case of the space-time fabric, object is assumed to follow the negative curvature. So it appears that the 
space-time fabric is another name for medium density.

The Universal Relativity (URL) [5, 4] takes into account the fact that the light is not relative, and the speed of the light is a universal constant that is determined by the electric properties of the medium. In the Universal Relativity, the time is absolute. The mass of an object is absolute. A moving body contracts in all directions leading to a volume contraction and hence mass density dilation while the mass remains unchanged. The mass density dilation leads to the surface gravity dilation. When a speed of a moving object reaches the speed of light, the volume of the object becomes zero, and in turn, the mass density will become infinite turning itself in to a transient black hole with infinite surface gravity; this prevents an object ever reaching the speed of light, the natural speed limit of any object.

The General Universal Relativity (GURL) extends the Universal Relativity (URL) derived for an inertial frame moving at constant speed to a frame moving at an accelerated speed. As in the case of inertial frames moving at constant speed, time and mass are absolute for accelerating bodies. The time and mass do not depend on the state of the object whether it is at stand still, moving at a constant speed or at an accelerated rate. Unlike on an inertial frame, the volume contraction on accelerating frame depends on time and acceleration; the volume contraction is a nonlinear function of time and acceleration. As time reaches $t=(c-u) / \alpha$, the acceleration body reaches zero volume and infinite mass density, turning itself into a transient black hole. When acceleration is zero, the General Universal Relativity (GURL) is exactly equal to the Universal Relativity (URL) of an inertial frame.

How in the universe one came to a preposterous conclusion that the universe is expanding based on the Hubble's observation that the nearby galaxies are moving away from us? Is it any different from if a cave-man has come out of his burrow for the first time and seen an object (perhaps, an air plane) moving in space, and declaring on the spot that the space is expanding? Yet, even in this era of scientific discovery and advancement, people still seem to believe the nonsense that the space is expanding. How can the space expand? The space is a "non-thing". Only "things expand or contract". Are we too suffering from "the cave-man's syndrome"? If you are trying to justify the claim that the universe is expanding by using the General Relativity as a theoretical basis, you are still living in the ancient time; the General Relativity does not hold true [4]. If you say universe is expanding when you see nearby galaxies are moving away, what do you say when you encounter a galaxy that is moving towards you? Are you going to say universe is contracting? Surely, expansion and contraction of the space can't take place at the same time. Further expansion or contraction can't be galaxy dependent. With the space expansion theory, there are three alternatives all the galaxies must follow; all the galaxies move away from us, all the galaxies move toward us, or all the galaxies remain stand still to us. The galaxies have no independent choice; it is all or none. With the space expansion theory, one galaxy can't move toward us or another galaxy can't remain at stand still without radial movement; all the galaxies must move away from us at a speed determine by the space expansion speed. What if two galaxies at equal distance from us move away at different speeds; how do you explain that using the space expansion theory? We already know at least one galaxy, Andromeda Galaxy [10], is moving toward us. There may be more galaxies that are moving toward us. There may be galaxies that we see no radial movement at all. The expanding space idea does not make any sense at all. The universe is not expanding. The space is not expanding. The galactic red-shift we observe on earth is an indication that the galactic orbits are dilating; every galaxy has its own orbit. The galactic orbits dilate when there is increase of galactic masses. Whether an orbit of a galaxy dilate, contract, or remains unchanged, our observation of it, that is whether it is moving away from us, moving toward us, or remains stand still depend on our location.

If the Hubble relationship holds true a big bang is not possible, and the age of the universe would be unbounded or infinite.

The galactic red shift is a result of the observer dependent red shift due to the radial galactic movement as well as the observer independent permanent red shift due to the propagation loss. The further the galaxy away the larger the propagation loss and hence the larger the red shift. The permanent red shift limits our visible universe. The limit of the visible universe does not indicate a beginning of the universe. However, information beyond the visible region can still be obtained in the spectrum below the visible region of the spectrum, or in the microwave background.

For all the objects in space to be free and independent, each object must have its own speed and its own orbit; galaxies are no exception. As such, for all the galaxies to be free and independent, each galaxy must be moving on its own orbit at an appropriate speed. Speed is the currency in the universe. Any object in space that is stand still will be swallowed up by the nearest object with higher gravity; it is no different from the dynamics of the present day corporations where a small cash-strapped company will be gobbled up by a bigger company with cash. From the Hubble's $[8,10]$ observation, It is clear that the nearby galaxies are moving away from us. We also know, if the nearby galaxies are orbiting around the Milky Way galaxy, the nearby galaxies will move away at a radial velocity at an accelerated rate when the mass of the nearby galaxies increase. What does that indicate? It indicates that the Milky Way galaxy is at the center of a Local Galactic Cluster (LGC) where the rest of the galaxies in the cluster are orbiting around.

In the universe, each galaxy belongs to a Local Galactic Cluster (LGC). Each Local Galactic Cluster (LGC) has an orbiting center where the rest of the galaxies in the cluster are orbiting around. Each local cluster must be orbiting around even a bigger cluster. In our cluster of galaxies, it happens to be us, the Milky Way Galaxy, that the rest of the galaxies in the Local Galactic Cluster are orbiting around. This is exactly why we see what we see when we point our telescopes to nearby galaxies. We see some of the nearby galaxies are moving away from us simply because we are in a galaxy at the orbiting center, and nearby galactic orbits are dilating due to the increase of masses of the galaxies. Any nearby galaxy experiencing mass reduction will be moving toward us at a 
radial speed due to orbit contraction since we are located in a galaxy at the orbiting center of a local cluster. Theoretically, the radial movement of the galaxies due to change of mass always takes place at an accelerated rate; this agrees with the observation data too, because again and again it has been observed that the nearby galaxies are moving away at an accelerated rate. The galaxies in a galactic cluster can also move away from the orbiting center when the mass of the center of the galactic cluster, in our case the mass of the Milky Way galaxy, is increasing too. We see that the nearby galaxies are moving away from us because the masses of those galaxies in the Milky Way Local Galactic Cluster (MW-LGC) are increasing. The Andromeda Galaxy is moving toward us because its mass is decreasing. The universe is not running away; the space is not running away; what is taking place is orbit fine tuning due to the changes in mass.

A Local Galactic Cluster (LGC) also has the property that if the diameter of a Local Galactic Cluster is $R$, then $R<c / H_{\max }$, where $H_{\max }$ is the largest $H_{i}, \forall i=1,2,3, \ldots n-1$. This property prevents the radial speed of any galaxy ever exceeding the speed of light, i.e. $u<c$. All the galaxies within any cluster are within the radius $c / H_{\max }$. Any galaxy outside this radius will be a member of a different Local Galactic Cluster.

Although we are not at the center of the universe, we are at the center of a local galactic cluster. There must be blue planets that support life at the centers of every Local Galactic Cluster. The poor utilization of the resources as well as the reality that one species has to consume another for life to exist indicate that the universe is not a work of a grand designer; it is a bad design in every sense; a material wasteland (wasted-universe) and cruelty at its extreme for survival.

When we point our telescopes into the distance, what we see is not our own past, but our distant neighbors'. When we measure deep space temperature, what we get is just that, not a snap shot of a baby universe. When we are dealing with extremely small quantities or extremely large quantities, it is possible to make use of the data to justify whatever we want to rather than what it really is; after all didn't we carry a clock in an air plane around the world to justify that the time is relative, a false concept to begin with, even though the difference is considered to be few billionth of a second. Contrary to those experiment results, now we know time is absolute, not relative. If we forget this simple fact, we are creating a dreamed up imaginary universe. Multi-verses (a load of crap in a glittery wrap to go), bubble universes (bubbla-rama, indigestible Soviet made Swiss-cheese), membrane collisions (Professor's stroke), inflation (royal-crap, Santa clause just keeps giving, more than six billion households in a fractions of a second in new year's eve, where does Santa get all those toys?), collapsing wave function universe (since there is no wave-particle duality [6], validity of wave function is questionable), non-collapsing wave function multi-verse (la la land ... where every dream come true ... you can really dream big here ...), dead and alive infinite copies (nonsense, way to sell million copies is to mystify it; they will buy), curled up dimensions (couch in front of TV), deep space temperature map representation of a baby universe (a lullaby, once upon a time universe was a baby...Good Night Moon ... Good Night Baby-Universe ...), distant entanglement (Houdini-fication), brain and neural entanglement (nonsense), teleportation by entanglement (science fiction), dark matter (sciencing in the dark ... what a feeling ...), dark energy (on voodoo practitioner's $8^{\text {th }}$ ball), anti-matter (the origin of anti-matter, the Dirac equations, does not hold true when special relativity does not hold true; what is an anti-neutron? do neutrons and anti-neutrons annihilate?), positron (just holes), negative energy (the attractive force between two extremely close plates is due to gravity; it is not due to some mysterious negative energy as it is proclaimed to be) and many more are simply a response to "publish or perish" rather than a real solution to anything; they are as bizarre as the concept of expanding space. Unlike the real science, the voodoo-fied or Houdini-fied science sell millions of copies; good business.

The universe is not expanding, space is not expanding, and a burst of light cannot travel forever; a burst of light will be frequency-shifted out of the visible region as it travel greater and greater distances due to path energy loss, and finally the burst will succumb to it; otherwise, the universe would have been a glowing place. There is/was no big-bang. Contrary to those misguided-Pundits who claim to know exact percentage of total mass, total energy, and everything in the universe, the amount of mass and energy in the universe is not calculable or knowable; there is no dark energy, and no dark matter in the universe, only the miss-understood darkness.

There is a maximum distance a burst of light can travel and it is determined by the path degradation of the medium. Unlike the Energizer bunny that keeps going and going, a burst of light does not keep going and going forever within the visible region of the frequency spectrum, and it is not expected to travel forever. The range or the maximum distance any light burst can travel without being frequency shifted out of the visible region of the spectrum imposes a limit to how far we can see in the space or the visible universe. The horizon of the visible universe is observer dependent; it moves when observer moves. To claim that we can't see beyond a certain point in space because we have reached the origin of the universe is simply preposterous. The fact that the visible universe horizon moves with the observer, or observer dependent, is an indication that the size of the universe is not finite. The light bursts that get frequency shifted out of the visible region of the spectrum due to path deterioration represent the cosmic microwave background; that is what a deep space temperature maps are; they provide us the information about the makeup of the universe outside the our visible horizon; however, the same information is available within the visible region for someone elsewhere at a distance due to moving visible horizon.

Houston, we have a problem; the light is not relative, gravity does not bend light, frequency is quantized, light spectrum is discrete, there is a maximum distance light can travel, visible universe is an observer dependent moving horizon and hence the universe is not finite, cosmic microwave background is not an indication universe had an origin, amount of matter and energy in the universe is not calculable, there is no dark matter, there is no dark energy, gravity and acceleration are not equivalent, time is absolute, mass is absolute, no relativistic-mass, no space-time, functionally the so-called space-time fabric is just the same as 
medium density, no random light particles or photons, particles are not waves and waves are not particles, no fourth dimension, the universe is neither expanding nor contracting and as a result no big-bang (a big-nonsense), nothing in nature is probabilistic, probability is a human invention, a collision with an asteroid brings ice-age, and there may be a blue planet at the orbiting center of each Local Galactic Cluster (LGC). The universe is not a product of a grand designer. We may not be alone. If you want to find a blue planet that support life you are looking at wrong places, look at the centers of Local Galactic Clusters (LGC). Every Local Galactic Cluster (LGC) should harbor a blue planet that supports life in the galaxy at its orbiting center. There is plenty of Local Galactic Clusters (LGC) in the universe.

The red shift of light is a result of observer dependent galactic radial movement as well as the observer independent permanent red shift due to propagation loss. The galactic radial movement is just that, a galactic radial movement; not a space expansion. If you misinterpret this fact, you will be sinking deep inside an inflationary puddle, the quick sand.

\section{Appendix A: The Effect of Acceleration on Lateral Dimensions}

Consider a cylindrical cabin (Figure 1and 2, Section 2). Now, assume at time $\mathrm{t}=\tau$, a light pulse is fired vertically from $P$ to $Q$. When the cabin is at stand still (Figure 1, Section 2), the light pulse travels straight vertically from $P$ to $Q$ with respect to an observer inside the cabin as well as an observer outside the cabin. So the time $T$ taken for the light pulse to travel from $P$ to $Q$ is given by,

$$
\mathrm{T}=\mathrm{H} / \mathrm{c}
$$

where $c$ is the speed of light.

Since the light is not relative and time is absolute, $T$ is the time taken for the light pulse to travel from $P$ to $Q$ with respect to any observer inside or outside the cabin irrespective of whether the cabin is at stand still or moving. It doesn't matter whether the cabin is moving or at stand still, an outside observer always sees that the light pulse travel straight up vertically at speed $c$, the speed of light. The light pulse has no momentum and hence does not follow the cabin; what an outside observer sees is a light pulse that keeps bouncing up and down. Once the light pulse is out of the source, it has nothing to do with the source, and the density gradient of the medium determine where the light pulse goes.

Now, let us consider the accelerating cabin (Figure 2, Section 2). With respect to a stationary outside observer, the light pulse travel vertically from $P$ to $Q$, and takes time $T$. However, for an observer inside the cabin, it is a completely different story. Since the observer inside the cabin is moving horizontally at an acceleration $\alpha$ while the light pulse is moving vertically up perpendicular to the direction motion of the cabin, the light pulse takes parabolic path with respect to the observer inside the cabin as shown in Figure 2, Section 2. With respect to an observer inside the cabin, the light travels at speed $c$ on a parabolic path PQ'. Consider a small section RS that the light pulse travel in time interval $\Delta \mathrm{t}$ [Figure 5].

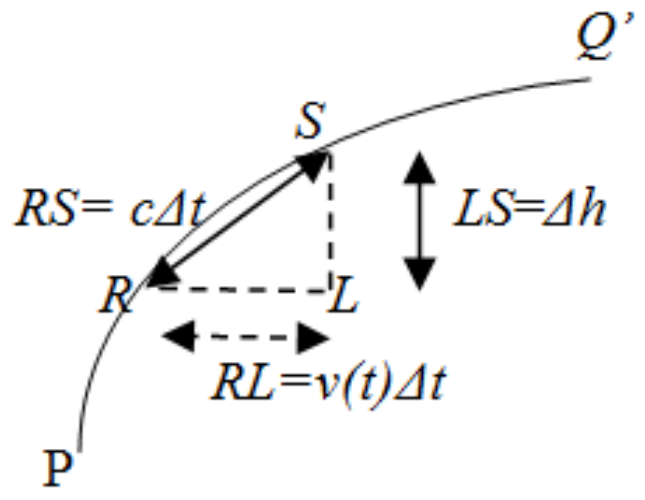

Figure 5. At interval on the parabolic path $P Q$ '.

The $\Delta t$ is chosen to be so small that $v(t)$ can be considered to remain constant during the time interval $\Delta t$. During the time interval $\Delta t$, the light travels a distance $c \Delta t$ from $R$ to $S$ on a curved parabolic path. For the same duration $\Delta t$, the cabin travels horizontally a distance of $v(t) \Delta t$. Therefore, with respect to an observer inside the cabin the light pulse travels $R L=v(t) \Delta t$ horizontally during the time duration $\Delta t$. So, with respect to an observer inside the accelerating cabin, the light pulse travels a vertical distance of $L S=\Delta h$. We can make the time duration $\Delta t$ as small as possible so that, RLS is a right angle triangle. So, the $\Delta h$ is given by,

$$
\begin{aligned}
& \Delta \mathrm{h}=\left[(\mathrm{c} \Delta \mathrm{t})^{2}-(\mathrm{v}(\mathrm{t}) \Delta \mathrm{t})^{2}\right)^{1 / 2} \\
& \Delta \mathrm{h} / \Delta \mathrm{t}=\mathrm{c}\left[1-(\mathrm{v}(\mathrm{t}) / \mathrm{c})^{2}\right]^{1 / 2}
\end{aligned}
$$

Since $\lim _{\Delta t \rightarrow 0} \Delta h / \Delta t=\partial h / \partial t$, we get,

$$
\begin{aligned}
\partial \mathrm{h} / \partial \mathrm{t} & =\mathrm{c}\left[1-(\mathrm{v}(\mathrm{t}) / \mathrm{c})^{2}\right]^{1 / 2} \\
\partial \mathrm{h} & =\mathrm{c}\left[1-(\mathrm{v}(\mathrm{t}) / \mathrm{c})^{2}\right]^{1 / 2} \partial \mathrm{t}
\end{aligned}
$$

We know that it takes time $T$ for the light pulse to travel on a straight path from $P$ to $Q$ when the cabin is at stand still (not moving). It also takes the same time $T$ for the light pulse to travel from $P$ to $Q$ with respect to a standstill observer outside the cabin when the cabin is moving. Since the time is absolute, it also takes time $T$ for the light pulse to travel a parabolic path from $P$ to $Q$ ' with respect to an observer inside the accelerating cabin. In other words, since the time is absolute, the time taken for the pulse to hit the ceiling is always $T$ irrespective of whether the cabin is moving at a constant speed, accelerating, or at standstill; it doesn't matter whether the observer is inside the cabin or outside the cabin. This is true because the propagation of light is not relative. The time it takes for a pulse of light to travel from point $P$ to point $Q$ does not depend on the observer, it doesn't matter where the observer is or what the observer doing.

So, we have the height of the accelerating cabin at time $\tau$,

$$
\mathrm{h}=\int_{p}^{q} c\left[1-(\mathrm{v}(\mathrm{t}) / \mathrm{c})^{2}\right]^{1 / 2} \partial \mathrm{t}
$$

where $p=\tau$, and $q=\tau+T$. We use the approximation,

So, we have,

$$
(1-\mathrm{x})^{1 / 2} \cong 1-\frac{1}{2} \mathrm{x} \text { for } x<<1 \text {. }
$$




$$
\mathrm{h}=\int_{p}^{q} c\left[1-\frac{1}{2}(\mathrm{v}(\mathrm{t}) / \mathrm{c})^{2}\right] \partial \mathrm{t}
$$

Since, $v(t)=u+\alpha t$, we have,

$$
v(t)=u(1+\alpha t / u)
$$

Substituting for $v(t)$ from (A8) in (A7), we get,

$$
\begin{gathered}
\mathrm{h}=\int_{p}^{q} c\left\{1-\frac{1}{2}(\mathrm{u} / \mathrm{c})^{2}\left[1+2 \alpha \mathrm{t} / \mathrm{u}+(\alpha \mathrm{t} / \mathrm{u})^{2}\right]\right\} \partial \mathrm{t} \\
\mathrm{h}=\mathrm{c}\left\{\mathrm{t}-\frac{1}{2}(\mathrm{u} / \mathrm{c})^{2}\left[\mathrm{t}+(\alpha / \mathrm{u}) \mathrm{t}^{2}+\frac{1}{3}(\alpha / \mathrm{u})^{2} \mathrm{t}^{3}\right]\right\} \tau_{\tau}^{\tau+\mathrm{T}} \\
\mathrm{h}=\mathrm{c}\left\{\mathrm{T}-\frac{1}{2}(\mathrm{u} / \mathrm{c})^{2}\left[\mathrm{~T}+(\alpha / \mathrm{u})\left((\tau+\mathrm{T})^{2}-\tau^{2}\right)+\frac{1}{3}(\alpha / \mathrm{u})^{2}\left((\tau+\mathrm{T})^{3}-\tau^{3}\right)\right]\right\} \\
\mathrm{h}=\mathrm{c}\left\{\mathrm{T}-\frac{1}{2}(\mathrm{u} / \mathrm{c})^{2}[\mathrm{~T}+(\alpha \mathrm{T} / \mathrm{u})(2 \tau+\mathrm{T})+\right. \\
\left.\left.\frac{1}{3}(\alpha / \mathrm{u})^{2} \mathrm{~T}\left((\tau+\mathrm{T})^{2}+\tau(\tau+\mathrm{T})+\tau^{2}\right)\right]\right\} \\
\mathrm{h}=\mathrm{cT}\left\{1-\frac{1}{2}(\mathrm{u} / \mathrm{c})^{2}[1+(\alpha / \mathrm{u})(2 \tau+\mathrm{T})+\right. \\
\left.\left.\frac{1}{3}(\alpha / \mathrm{u})^{2}\left((\tau+\mathrm{T})^{2}+\tau(\tau+\mathrm{T})+\tau^{2}\right)\right]\right\} \\
\mathrm{h}=\mathrm{cT}\left\{1-\frac{1}{2}(\mathrm{u} / \mathrm{c})^{2}[1+(\alpha \tau / \mathrm{u})((1+\mathrm{T} / \tau)+1)+\right. \\
\left.\left.\frac{1}{3}(\alpha \tau / \mathrm{u})^{2}\left((1+\mathrm{T} / \tau)^{2}+(1+\mathrm{T} / \tau)+1\right)\right]\right\}
\end{gathered}
$$

$\mathrm{h}=\mathrm{cT}\left\{1-\frac{1}{2}(\mathrm{u} / \mathrm{c})^{2}\left[1+(\alpha \tau / \mathrm{u})(2+\mathrm{T} / \tau)+(\alpha \tau / \mathrm{u})^{2}\left(1+\mathrm{T} / \tau+\frac{1}{3}(\mathrm{~T} / \tau)^{2}\right)\right]\right\}$.

Eqn. (A1) gives, $T=H / c$. Further, the choice of $\tau$ is arbitrary. It can be any time $t$. Therefore, we can replace $\tau$ with $t$.

Now, substituting for $T$ and replacing $\tau$ with $t$ we get,

$$
\begin{gathered}
\mathrm{h}=\left[1-\frac{1}{2} \beta(\mathrm{t})(\mathrm{u} / \mathrm{c})^{2}\right] \mathrm{H}, \\
\beta(\mathrm{t})=\left\{1+(\alpha \mathrm{t} / \mathrm{u})[2+\mathrm{H} / \mathrm{ct}]+(\alpha \mathrm{t} / \mathrm{u})^{2}\left[1+\mathrm{H} / \mathrm{ct}+\frac{1}{3}(\mathrm{H} / \mathrm{ct})^{2}\right]\right\} .
\end{gathered}
$$

Earlier we used the approximation, $(1-\mathrm{x})^{1 / 2} \cong 1-\frac{1}{2} \mathrm{x}$ for $\mathrm{x}<<1$. Now we use it in reverse direction on eqn. (A11), to obtain,

$$
\mathrm{h}=\left[1-\beta(\mathrm{t})(\mathrm{u} / \mathrm{c})^{2}\right]^{1 / 2} \mathrm{H}
$$

Let, $\eta(t)=\left[1-\beta(t)(u / c)^{2}\right]^{1 / 2}$, then, we get,

$$
\mathrm{h}=\mathrm{n}(\mathrm{t}) \mathrm{H}
$$

where $\eta(t)$ is a function of inertial speed $u$, acceleration $\alpha$, the speed of light $c$, and time $t . \eta(t)$ is the Universal Lateral Contraction Factor along the direction perpendicular to the direction of motion at time $t$. Now, we can summarize the main result in the General Lateral Contraction Theorem 3.1 that is given above in Section 3.

\section{Appendix-B: The Longitudinal Dimension of an Accelerating Object}

Here, we use the same cylindrical cabin. Now, we fire a light pulse from $P$ to $Q$ at time $\tau$ in the direction of motion horizontally. There is a reflector at $Q$. So the light pulse will reflect at $Q$ and travel back from $Q$ to $P$.

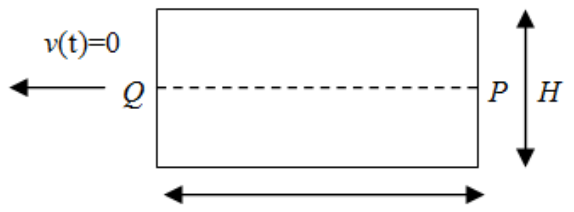

$L$

Figure 6. Cylindrical cabin at rest.

Let the forward time, $t_{f}=$ the time taken for the light pulse to travel from $P$ to $Q$, and the backward time, $t_{b}=$ the time taken for the light pulse to travel from $Q$ to $P$.

So, the round trip time $T_{o}$ is given by,

$$
\mathrm{T}_{\mathrm{o}}=\mathrm{t}_{\mathrm{f}}+\mathrm{t}_{\mathrm{b}} \text {. }
$$

When the cabin is at standstill, the forward time, $t_{f}$ is the same as the backward time $t_{b}$. Therefore, we get,

$$
\mathrm{T}_{\mathrm{o}}=2 \mathrm{~L} / \mathrm{c}
$$

Although the forward time and the backward time are the same with respect to an observer inside the cabin when the cabin is at rest, it is no longer the case when the cabin is in motion. Let us consider the cabin in motion.

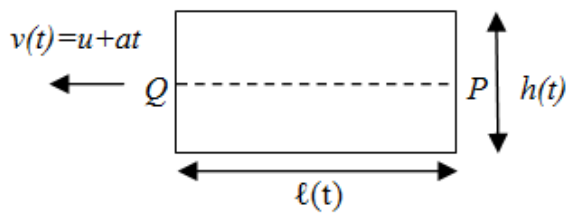

Figure 7. Accelerating Cylindrical Cabin.

We already know the lateral distance $h(t)$ from the previous section. We want to find the longitudinal distance $\ell(t)$ of the accelerating cabin. To do that, we fire a light pulse from $P$ toward $Q$. The light pulse will reflect back at $Q$. As we did for the case of stationary cabin, we want to find the return time of the light pulse, $T_{r}$. Since the forward time of the light pulse is $t_{f}$ and the backward time of the light pulse is $t_{b}$, we have,

$$
\mathrm{T}_{\mathrm{r}}=\mathrm{t}_{\mathrm{f}}+\mathrm{t}_{\mathrm{b}} \text {. }
$$

Here, the cabin is in motion, so, $t_{f} \neq t_{b}$. We have to find $t_{f}$ and $t_{b}$ separately.

Since the time is absolute, return time of the light pulse for a cabin at stand still must be the same as the return time of the light pulse for a moving cabin. The return time of a light pulse is independent of the state of the cabin. The cabin can be at stand still, moving at a constant speed, or accelerating, but the return time of a light pulse remains the same. In other words, $T_{r}=T_{o}$. So, we get,

$$
\mathrm{t}_{\mathrm{f}}+\mathrm{t}_{\mathrm{b}}=2 \mathrm{~L} / \mathrm{c}
$$

Let us assume that we fire a light pulse at $t=\tau$. Then, the distance light pulse travel from $P$ to reach $Q$ is given by,

$$
\ell(\mathrm{t})+\int_{\mathrm{p}}^{\mathrm{q}} \mathrm{v}(\mathrm{t}) \partial \mathrm{t}
$$

where, $p=\tau$, and $q=\tau+t_{f}$. Since the light pulse travel at the 
speed $c$ for time $t_{f}$ to reach $Q$ from $P$, we have the relationship,

$$
\ell(\mathrm{t})+\int_{\mathrm{p}}^{\mathrm{q}} \mathrm{v}(\mathrm{t}) \partial \mathrm{t}=\mathrm{ct}_{\mathrm{f}}
$$

Since $v(t)=u+\alpha t$, Substituting for $v(t)$, we have,

$$
\begin{gathered}
\ell(\mathrm{t})+\int_{p}^{q}(\mathrm{u}+\alpha \mathrm{t}) \partial \mathrm{t}=\mathrm{ct}_{\mathrm{f}} \\
\ell(\mathrm{t})+\left.\left[\mathrm{ut}+\frac{1}{2} \alpha \mathrm{t}^{2}\right]\right|_{\mathrm{p}} ^{\mathrm{q}}=\mathrm{ct}_{\mathrm{f}} \\
\ell(\mathrm{t})+\mathrm{ut}_{\mathrm{f}}+\frac{1}{2} \alpha\left[\left(\tau+\mathrm{t}_{\mathrm{f}}\right)^{2}-\tau^{2}\right]=\mathrm{ct}_{\mathrm{f}} \\
\ell(\mathrm{t})+\mathrm{ut}_{\mathrm{f}}+\frac{1}{2} \alpha \mathrm{t}_{\mathrm{f}}\left(2 \tau+\mathrm{t}_{\mathrm{f}}\right)=\mathrm{ct}_{\mathrm{f}} \\
\ell(\mathrm{t})+\frac{1}{2} \alpha \mathrm{t}_{\mathrm{f}}^{2}=[\mathrm{c}-(\mathrm{u}+\alpha \tau)] \mathrm{t}_{\mathrm{f}}
\end{gathered}
$$

Now, let us consider the return trip of the light pulse from $Q$ to $P$. The distance the light pulse has to travel to go from $Q$ to $P$ is given by,

$$
\ell(\mathrm{t})-\int_{\mathrm{i}}^{\mathrm{j}} \mathrm{v}(\mathrm{t}) \partial \mathrm{t}
$$

where, $i=\tau+t_{f}$, and $j=\tau+t_{f}+t_{b}$. Substituting for $v(t)$, we get,

$$
\begin{aligned}
& \ell(\mathrm{t})-\int_{i}^{j}(\mathrm{u}+\alpha \mathrm{t}) \partial \mathrm{t}=\mathrm{ct}_{\mathrm{b}} \\
& \ell(\mathrm{t})-\left[\mathrm{ut}+\frac{1}{2} \alpha \mathrm{t}^{2}\right]_{\mathrm{i}}^{\mathrm{j}}=\mathrm{ct}_{\mathrm{b}}
\end{aligned}
$$

From eqn. (B3), we have $t_{f}+t_{b}=2 L / c$, and hence,

$$
\begin{gathered}
\ell(\mathrm{t})-\left\{\mathrm{ut}_{\mathrm{b}}+\frac{1}{2} \alpha\left[(\tau+2 \mathrm{~L} / \mathrm{c})^{2}-\left(\tau+2 \mathrm{~L} / \mathrm{c}-\mathrm{t}_{\mathrm{b}}\right)^{2}\right]\right\}=\mathrm{ct}_{\mathrm{b}} \\
\ell(\mathrm{t})-\left\{\mathrm{ut}_{\mathrm{b}}+\frac{1}{2} \alpha \mathrm{t}_{\mathrm{b}}\left[2(\tau+2 \mathrm{~L} / \mathrm{c})-\mathrm{t}_{\mathrm{b}}\right]\right\}=\mathrm{ct}_{\mathrm{b}} \\
\left.\ell(\mathrm{t})-\mathrm{ut}_{\mathrm{b}}-\mathrm{ct}_{\mathrm{b}}-\alpha \mathrm{t}_{\mathrm{b}}(\tau+2 \mathrm{~L} / \mathrm{c})+\frac{1}{2} \alpha \mathrm{t}_{\mathrm{b}}{ }^{2}\right)=0 \\
\frac{1}{2} \alpha \mathrm{t}_{\mathrm{b}}{ }^{2}-(\mathrm{c}+\mathrm{u}+\alpha \tau) \mathrm{t}_{\mathrm{b}}+\ell(\mathrm{t})=2(\alpha \mathrm{L} / \mathrm{c}) \mathrm{t}_{\mathrm{b}}
\end{gathered}
$$

From eqn. (B6), we have,

$$
\frac{1}{2} \alpha \mathrm{t}_{\mathrm{f}}^{2}-(\mathrm{c}-\mathrm{u}-\alpha \tau) \mathrm{t}_{\mathrm{f}}+\ell(\mathrm{t})=0
$$

Subtracting, Eqn. (B10) from Eqn. (B9), and substituting for $t_{f}+t_{b}$, we get,

$$
\begin{gathered}
(\alpha \mathrm{L} / \mathrm{c})\left(\mathrm{t}_{\mathrm{b}}-\mathrm{t}_{\mathrm{f}}\right)+(\mathrm{c}-\mathrm{u}-\alpha \tau) \mathrm{t}_{\mathrm{f}}-(\mathrm{c}+\mathrm{u}+\alpha \tau) \mathrm{t}_{\mathrm{b}}=2(\alpha \mathrm{L} / \mathrm{c}) \mathrm{t}_{\mathrm{b}} \\
(\mathrm{c}-\mathrm{u}-\alpha \tau) \mathrm{t}_{\mathrm{f}}-(\mathrm{c}+\mathrm{u}+\alpha \tau) \mathrm{t}_{\mathrm{b}}=(\alpha \mathrm{L} / \mathrm{c})\left(\mathrm{t}_{\mathrm{f}}+\mathrm{t}_{\mathrm{b}}\right)
\end{gathered}
$$

Since $t_{f}+t_{b}=2 L / c$, we get,

$$
(c-u-\alpha \tau) t_{f}-(c+u+\alpha \tau) t_{b}=2 \alpha(L / c)^{2}
$$

From eqn. (B3), we have,

$$
\mathrm{t}_{\mathrm{f}}+\mathrm{t}_{\mathrm{b}}=2 \mathrm{~L} / \mathrm{c}
$$

Multiplying eqn. (B12) by $(\mathrm{c}+\mathrm{u}+\alpha \tau)$, we get,

$$
(\mathrm{c}+\mathrm{u}+\alpha \tau) \mathrm{t}_{\mathrm{f}}+(\mathrm{c}+\mathrm{u}+\alpha \tau) \mathrm{t}_{\mathrm{b}}=2(\mathrm{~L} / \mathrm{c})(\mathrm{c}+\mathrm{u}+\alpha \tau)
$$

Adding eqn. (B13) from eqn. (B11), we get,

$$
\begin{gathered}
\mathrm{ct}_{\mathrm{f}}=(\mathrm{L} / \mathrm{c})(\mathrm{c}+\mathrm{u}+\alpha \tau)+\alpha(\mathrm{L} / \mathrm{c})^{2} \\
\mathrm{t}_{\mathrm{f}}=\left(\mathrm{L} / \mathrm{c}^{2}\right)(\mathrm{c}+\mathrm{u}+\alpha \tau)+(\alpha / \mathrm{c})(\mathrm{L} / \mathrm{c})^{2} \\
\mathrm{t}_{\mathrm{f}}=\left(\mathrm{L} / \mathrm{c}^{2}\right)(\mathrm{c}+\mathrm{u}+\alpha \tau)[1+\alpha \mathrm{L} / \mathrm{c}(\mathrm{c}+\mathrm{u}+\alpha \tau)]
\end{gathered}
$$

From eqn. $(\mathrm{B} 6), \ell(\mathrm{t})=(\mathrm{c}-\mathrm{u}-\alpha \tau) \mathrm{t}_{\mathrm{f}}-\frac{1}{2} \alpha \mathrm{t}_{\mathrm{f}}^{2}$

$$
\ell(\mathrm{t})=(\mathrm{c}-\mathrm{u}-\alpha \tau) \mathrm{t}_{\mathrm{f}}\left[1-\frac{1}{2} \alpha \mathrm{t}_{\mathrm{f}} /(\mathrm{c}-\mathrm{u}-\alpha \tau)\right]
$$

Let $\mathrm{w}=\mathrm{u}+\alpha \tau$, then, $\mathrm{t}_{\mathrm{f}}=\left(\mathrm{L} / \mathrm{c}^{2}\right)(\mathrm{c}+\mathrm{w})[1+\alpha \mathrm{L} / \mathrm{c}(\mathrm{c}+\mathrm{w})]$. Let, $\varphi=1+\alpha \mathrm{L} / \mathrm{c}(\mathrm{c}+\mathrm{w})$, then,

$$
\begin{aligned}
\mathrm{t}_{\mathrm{f}} & =\left(\mathrm{L} / \mathrm{c}^{2}\right)(\mathrm{c}+\mathrm{w}) \varphi \\
\ell(\mathrm{t}) & =(\mathrm{c}-\mathrm{w}) \mathrm{t}_{\mathrm{f}}\left[1-\frac{1}{2} \alpha \mathrm{t}_{\mathrm{f}} /(\mathrm{c}-\mathrm{w})\right]
\end{aligned}
$$

Substituting for $t_{f}$ from eqn. (B16) in eqn. (B17), we get,

$$
\begin{aligned}
& \ell(\mathrm{t})=\left(\mathrm{L} / \mathrm{c}^{2}\right)\left(\mathrm{c}^{2}-\mathrm{w}^{2}\right) \varphi\left\{1-\frac{1}{2}\left[\alpha \mathrm{L}(\mathrm{c}+\mathrm{w}) /(\mathrm{c}-\mathrm{w}) \mathrm{c}^{2}\right] \varphi\right\} \\
& \ell(\mathrm{t})=\mathrm{L}\left[1-(\mathrm{w} / \mathrm{c})^{2}\right] \varphi\left\{1-\frac{1}{2} \varphi\left[\alpha \mathrm{L}(\mathrm{c}+\mathrm{w}) /(\mathrm{c}-\mathrm{w}) \mathrm{c}^{2}\right]\right\}
\end{aligned}
$$

Since $\tau$ is arbitrary, it can be any $t$. We can replace $\tau$ by $t$. Then, we have, $w=v$, and,

$$
\begin{gathered}
\ell(\mathrm{t})=\mathrm{L}\left[1-(\mathrm{v} / \mathrm{c})^{2}\right] \varphi\left\{1-\frac{1}{2} \varphi\left[\alpha \mathrm{L}(\mathrm{c}+\mathrm{v}) /(\mathrm{c}-\mathrm{v}) \mathrm{c}^{2}\right]\right\} \\
\varphi=1+\alpha \mathrm{L} / \mathrm{c}(\mathrm{c}+\mathrm{v}) .
\end{gathered}
$$

Let, $\gamma(\mathrm{t})=\varphi\left\{1-\frac{1}{2} \varphi\left[\alpha \mathrm{L}(\mathrm{c}+\mathrm{v}) /(\mathrm{c}-\mathrm{v}) \mathrm{c}^{2}\right]\right\}$.

Then, we get,

$\ell(\mathrm{t})=\left[1-(\mathrm{v} / \mathrm{c})^{2}\right] \gamma(\mathrm{t}) \mathrm{L}$,

where, $v=u+\alpha t$ and $u$ is the inertial speed, $\alpha$ is the acceleration, and $t$ is the time.

The longitudinal length of an accelerating body is a function the inertial speed $u$, acceleration $\alpha$, the speed of light $c$, and time $t$. Now, we can formulate the result in to the General Longitudinal Contraction Theorem 3.3 that is given above in Section 3.

Abstract in the Reference [4] should be read as "If the Maxwell's equations are relative, the relative Maxwell's equations also create Shear Electro-Magnetic (SEM) waves, which have a speed that depends on the speed of the inertial frame, and have much LOWER speed than the Transversal Electro-Magnetic (TEM) waves."

\section{References}

[1] Einstein, Albert, "On the Electrodynamics of Moving Bodies", Annalen der Physik, 17 (1905).

[2] Kennedy Robert, “A student Guide to Einstein's Major Papers”, Oxford University Press, 2012.

[3] Einstein, Albert, "Does the Inertia of a Body Depend Upon Its Energy Content?", Annalen der Physik, 18 (1905).

[4] Dahanayake, Bandula, "Maxwell's Equations and Propagation of Light: Not Relative", International Journal of Astrophysics and Space Science, Dec. 2015. 
[5] Dahanayake, Bandula, "Universal Relativity: Absolute Time and Mass", International Journal of Astrophysics and Space Science, Feb. 2015.

[6] Dahanayake, Bandula, "The Light in a New Light: Always a Wave, Never a Particle", Science Discovery, Aug. 2015.

[7] Dahanayake, Bandula, "Universe: Not Expanding", International Journal of Astrophysics and Space Science, Aug. 2014.
[8] Hubble Edwin, "A Relation between Distance and Radial Velocity among Extra-Galactic Nebulae”, Proc. N. A. S., 1929.

[9] Tegmark Max, "Our Mathematical Universe", Alfred A. Knoff, New York, 2014.

[10] Wikipedia. com, "Lorentz Transform, Special Relativity, General Relativity, Hubble's Law, Accelerating Universe, History of Big-Bang, Andromeda galaxy, Galaxy Clusters", Nov. 2015. 